\title{
Elasto-Dynamics of Quasicrystals
}

\author{
$\mathrm{Wu} \mathrm{Li}{ }^{1, *}$ and Tianyou Fan ${ }^{2}$ \\ 1 Institute of Science, Taiyuan University of Technology, Taiyuan 030024, China \\ 2 Department of Physics, Beijing Institute of Technology, Beijing 100081, China; tyfan2006@yeah.net \\ * Correspondence: liwu@tyut.edu.cn; Tel.: +86-0351-3176606; Fax: +86-0351-3176607 \\ Academic Editor: Enrique Maciá Barber \\ Received: 29 September 2016; Accepted: 11 November 2016; Published: 23 November 2016
}

\begin{abstract}
A review on elasto-dynamics of quasicrystals (QCs) and their applications based on mathematical elasticity is given. In this study, recent studies on elasto-dynamics of QCs are reviewed, in which the focus of the problem lies in the role of phason variables and the coupling effect between phonons and phasons in the dynamic deformation process. On summarizing and describing the development of the elastic dynamics of QCs, this review mainly presents theelasto-dynamics of QCs and their application in a variety of research areas, ranging from problems with different QCs, including one-, two-, and three-dimensional QCs to various coupling problems. The plane elasticity and anti-plane elasticity of quasicrystals are included in this review.
\end{abstract}

Keywords: quasicrystals; elasticity; dynamics; crack; dislocation

\section{Introduction}

Quasicrystals are viewed as a new structure of solids, different from crystals and possess non-crystallographic rotational symmetry and quasi-periodic long-range orientational symmetry [1]. After the discovery of quasicrystals, elasticity, dynamics of elasticity, defects and other subjects concerning the mechanical behavior of quasicrystalline materials provide many new challenges to researchers from a variety of areas [2-7]. Like other materials, quasicrystal is deformable under external loads and certain thermal loads. Regarding the elasticity of quasicrystals, theoretical physicists have provided various descriptions. Almost all of them regard the Landau density wave theory as the physical basis of the elasticity of quasicrystals [8-15].Inessence, the description of the elasticity of quasicrystals deemed that there are two displacement fields $\mathbf{u}$ and $\mathbf{w}$, in which the former $\mathbf{u}$ called the phonon field is similar to that in crystals; the latter $\mathbf{w}$ is called the phason field. They were first discussed by Bak [12,13] and Socalar et al. [14]. So the total displacement field in a quasicrystal can be expressed by $\overline{\mathbf{u}}=\mathbf{u} \| \oplus \mathbf{u}^{\perp}=\mathbf{u} \oplus \mathbf{w}$, in which $\mathbf{u}$ is in the parallel space, or the physical space; $\mathbf{w}$ is in the complement space, or the perpendicular space; which is an internal space and $\oplus$ denotes the direct sum. On the basis of the above physical framework and the extended methodology in mathematical physics from classical elasticity, the independent elastic constants for different symmetries of quasicrystals can be determined [16-21]. Then the mathematical elasticity theory of quasicrystals has been developed rapidly. In 2004, Fan and Mai give a review based on the static elasticity theory of various quasicrystals [22]. A monograph is devoted to the development of a mathematical elasticity theory of quasicrystals and its applications [23]. First, Levine and Lubensky et al. carried out extensive work in terms of the elasticity and dislocations in pentagonal and icosahedral quasicrystals, resulting in many solutions for the dislocations [24]. Then Li presented two solutions for a Griffith crack and a straight dislocation embedded in a decagonal quasicrystal $[25,26]$. Chen provided a three-dimensional elastic problem of one-dimensional hexagonal quasicrystal, and gave a general solution for this problem [27]. Meanwhile, Liu et al. obtained the general solutions and the governing equations for plane elasticity of one-dimensional quasicrystals [28]. Based on the stress potential function, Li used a complex function 
method to accomplish notch problem of two-dimensional quasicrystals [29]. Wang and Gao obtained some solutions for some defect problems of one-, two-dimensional quasicrystal [30-32]. Coddens discussed the elasticity and dynamics of the phason in quasicrystals [33]. Wang et al. discussed the phonon- and phason-type inclusions in icosahedral quasicrystals [34]. Guo et al. also discussed an elliptical inclusion in hexagonal quasicrystals [35]. More recently, the phonon-phason elasticity of QCs has attracted a lot of attention too. For example, Radi and Mariano have described linear elasticity of QCs, and obtained some meaningful results for the straight cracks and dislocations in two-dimensional quasicrytals [36-38]. An important reference concerning fundamental aspects of generalized elasticity and dislocation theory deriving the generalized three-dimensional elastic Green tensor and all the dislocation key-formulas including an application to dislocation loops for arbitrary quasicrytals is presented by Lazar and Agiasofitou [39]. Li provided fundamental solutions for thermo-elasticity based on half infinite plane cracks embedded in one-dimensional hexagonal QCs and some solutions for one-dimensional hexagonal QCs [40-42]. Of course, dislocation and plate theory of QCs have been developed by some researchers, for example, Sladek et al. [43], Li and Chai [44]. Because of the lack of data of constitutive equations about plastic deformation of quasicrystals, the related researches are fairly complicated. The existence of phason degrees of freedom leads to the essential difference between crystals and quasicrystals. Wollgarten et al. used a dislocation mechanism to study plastic deformation of quasicrystals [45]. A model of the plastic deformation of icosahedral quasicrystals was provided by Feuerbacher et al. [46] and refined by Messerschmidt et al. [47]. A mass of studies have been executed on the deformed Al-Pd-Mn single quasicrystals through a series of experimental observations [48-51].

As an important subject, dynamics of elasticity of quasicrystals is inevitable. Recent studies on the elasto-/hydro-dynamics of quasicrystals have made preliminary but significant progress. During the development of this study we describe different points of view on the role of the phason variables in the dynamic process of quasicrystals. We can primarily summarize the relevant viewpoints as follows: (1) The phason field $\mathbf{w}$ is similar to phonon field $\mathbf{u}$ in quasicrystals, represents long-wavelength propagation. This idea originated from Bak $[12,13]$ who indicated that the phason describes particular structure fluctuations (or structure disorders) in quasicrystals, and it can be viewed as a six-dimensional space description; (2) The phason field $\mathbf{w}$ is diffusion rather than wave propagation, so phasons play different roles to phonons in the hydrodynamics of quasicrystals. This idea can be found in Lubensky et al. [52], Francoual et al. [53] and Socolar et al. [54], etc. They claimed that the phason field $\mathbf{w}$ represents diffusion rather than wave propagation; thus, phasons play different roles than phonons in the hydrodynamics of QCs. The phason modes denote the relative motion of the density waves and the phason field $\mathbf{w}$ is not sensitive to spatial translations, such that the phasons are not oscillatory, instead, they are diffusive with very large diffusion times. Furthermore, some researchers explained that the motion of the phasons is atomic jumps. Following the above two models, Mikulla and Trebin et al. [55], Takeuchi et al. [56], Rösch [57] and co-workers carried out extensive work in terms of analytic and numerical methods, this results in many solutions in dislocation and crack dynamics for different quasicrystal systems. Similar to [58,59], Fan and his co-workers obtained some analytical solutions for some 1D and 2D quasicrystals [60-63]. More recently, Tupholme studied an anti-plane shear crack moving in one-dimensional hexagonal quasicrystals based on the reference [64,65]. Li gave a general solution for elasto-dynamics of two dimensional quasicrystals [66]. In contrast to the generalized elasto-dynamics, this kind of study may be named elasto-/hydro-dynamics of quasicrystals, because the equations of motion of phonons are elasto-dynamics equations, while the equations of motion of phasons are diffusion equations originated from the hydrodynamics; (3) There is a recent and promising model introduced by Agiasofitou and Lazar for describing the dynamics of quasicrystals, in which the authors clarify that an elasto-dynamic model of wave-telegraph type can be used [67]. Based on this model, phonons are represented by waves, and phasons by waves damped in time and propagating with finite velocity. Therefore, the equations of motion for the phonon fields are of wave type and for the phason fields are of telegraph type. The proposed model constitutes 
a unified theory in the sense that already established models in the literature can be recovered as asymptotic cases of it. Moreover, the same authors have investigated and compared three models of dynamics: the elasto-dynamic model of wave type and the elasto-hydrodynamic model and the elasto-dynamic model of wave-telegraph type [68]. For these models, they derived the equations of motion of dislocations for arbitrary quasicrystals. To the best of the author's knowledge these are the main-current models in the literature for the description of the dynamics of quasicrystals.

The different points of view on the dynamics of elasticity of quasicrystals lead to the difficulty of the study. Though some researchers believe that the hydrodynamics, i.e., the model (2) based on the viewpoint of Lubensky et al., is a fundamental standpoint, the theory has not been verified experimentally. In this case we give a general survey on the development of different theories and their applications below, which may be beneficial to readers who are interested in the dynamics of quasicrystals. This review is focused on the basic concepts and fundamental framework of the theory of elasto-dynamics for quasi-crystalline materials, including some 1D, 2D, and 3D quasicrystals. A decomposition and superposition procedure has been suggested to simplify the elasto-dynamics problems of quasi-crystals. Simplification of the basic equations provides the possibility to solve boundary value or initial-boundary value problems of elasto-dynamics elasticity. The above may be seen as a development of the theory and methodology to those of classical dynamic elasticity. Also, some elasto-dynamic problems for some 1Dand 2D quasi-crystals are studied, as are some examples of their theory.

\section{Elasto-Dynamics of Quasicrystals Followed Bak's Argument}

Ding et al. first proposed the elasticity of quasicrystals in [19]. The basic equations in deformation geometry are repressed by

$$
\varepsilon_{i j}=\frac{1}{2}\left(\frac{\partial u_{i}}{\partial x_{j}}+\frac{\partial u_{j}}{\partial x_{i}}\right), w_{i j}=\frac{\partial w_{i}}{\partial x_{j}}
$$

In linear elasticity of quasicrystals the stress tensors (generalized Hooke's law) related to the strain tensors can be expressed by

$$
\left\{\begin{array}{c}
\sigma_{i j}=C_{i j k l} \varepsilon_{k l}+R_{i j k l} w_{k l} \\
H_{i j}=K_{i j k l} w_{k l}+R_{k l i j} \varepsilon_{k l}
\end{array}\right.
$$

where $\sigma_{i j}$ and $\varepsilon_{i j}$ phonon stresses and strains, $u_{i}, w_{i}$ are phonon and phason displacements, $H_{i j}$ and $w_{i j}$ phason stresses and strains, $C_{i j k l}, K_{i j k l}$ and $R_{i j k l}$ the phonon, phason, phonon-phason coupling elastic constants respectively.

The law of the momentum conservation is assumed to hold for both the phonons and the phasons. For the elasto-dynamics, the viewpoint raised by Bak in linear and small deformation case the equations of motion are:

$$
\frac{\partial \sigma_{i j}}{\partial x_{j}}=\rho \frac{\partial^{2} u_{i}}{\partial t^{2}}, \frac{\partial H_{i j}}{\partial x_{j}}=\rho \frac{\partial^{2} w_{i}}{\partial t^{2}}
$$

where $\rho$ denotes the average mass density of the material. In this sense, Equation (3) follows Bak's argument [12,13]. The elasto-dynamics equations can be deduced by substituting (1) and (2) into (3). The mathematical structure of this theory is relatively simple, and the formulations are similar to that of classical elasto-dynamics; many researchers followed the formulation to develop the elasto-dynamics of quasicrystals and applications in defect dynamics and thermodynamics. In the following sections we give some examples of the theory. Some of them have been published. 


\subsection{Example 1: Basic Equations for Elasto-Dynamics of Anti-Plane Elasticity of Some Quasicrystals}

The basic equations of anti-plane elasticity for one-dimensional hexagonal quasicrystals or three-dimensional icosahedral, and cubic quasicrystals take a similar form. Let us consider icosahedral quasicrystals first. The stress tensors are related to the strain tensors repressed by

$$
\begin{gathered}
\sigma_{z y}=\sigma_{y z}=\mu \frac{\partial u_{z}}{\partial y}+R \frac{\partial w_{z}}{\partial y}, \sigma_{x z}=\sigma_{z x}=\mu \frac{\partial u_{z}}{\partial x}+R \frac{\partial w_{z}}{\partial x} \\
H_{z y}=\left(K_{1}-K_{2}\right) \frac{\partial w_{z}}{\partial y}+R \frac{\partial u_{z}}{\partial y}, H_{z x}=\left(K_{1}-K_{2}\right) \frac{\partial w_{z}}{\partial x}+R \frac{\partial u_{z}}{\partial x}
\end{gathered}
$$

Substituting (4) into (3) yields

$$
\mathbf{A} \nabla^{2} \mathbf{u}-\rho \frac{\partial^{2}}{\partial t^{2}} \mathbf{u}=0
$$

where $\nabla^{2}$ is two dimensional Laplace operator, $\rho$ is the density of the material, $\mathbf{u}=\left[u_{z}, w_{z}\right]^{\mathrm{T}}$ and $\mathbf{A}=\left[\begin{array}{cc}\mu & R \\ R & K_{1}-K_{2}\end{array}\right]$, " $\mathrm{T}$ " denotes the transpose of a matrix.

A simple approach for simplifying the Equation (5) is to introduce two new functions as follows

$$
\mathbf{u}=B \varphi
$$

where $\mathbf{B}=\left[\begin{array}{cc}\alpha & -R \\ R & \alpha\end{array}\right]$, and $\boldsymbol{\varphi}=[\phi, \psi]$.

Then the Equation (5) reduces to

$$
\mathbf{S} \nabla^{2} \boldsymbol{\varphi}=\frac{\partial^{2}}{\partial t^{2}} \boldsymbol{\varphi}
$$

where

$$
\begin{gathered}
\mathbf{S}=\left[\begin{array}{l}
s_{1}^{2} \\
s_{2}^{2}
\end{array}\right] \\
\alpha=\frac{1}{2}\left[\mu-\left(K_{1}-K_{2}\right)+\sqrt{\left(\mu-\left(K_{1}-K_{2}\right)\right)^{2}+4 R^{2}}\right] \\
s_{j}=\sqrt{\frac{\varepsilon_{j}}{\rho}}, j=1,2 \\
\varepsilon_{1,2}=\frac{1}{2}\left[\mu+\left(K_{1}-K_{2}\right) \pm \sqrt{\left(\mu-\left(K_{1}-K_{2}\right)\right)^{2}+4 R^{2}}\right]
\end{gathered}
$$

$s_{j}$ indicates the speeds of wave propagation. It is clear that the results are caused by the coupled phonon and phason fields. Suppose there is no couple effect, i.e., $R \rightarrow 0$, one can get

$$
s_{1} \rightarrow \sqrt{\frac{\mu}{\rho}}, s_{2} \rightarrow \sqrt{\frac{\left(K_{1}-K_{2}\right)}{\rho}}
$$

and the known constant $\sqrt{\frac{\mu}{\rho}}$ indicates the elastic wave speed of the phonon field, while $\sqrt{\frac{K_{1}-K_{2}}{\rho}}$ requires $K_{1}-K_{2}>0$ and represents the speed of the elastic wave speed of the phason field. 
Substituting (6) into (4) gets the displacement and stress expressions based on the two displacement functions $\phi$ and $\psi$

$$
\left\{\begin{array}{l}
\sigma_{y z}=\sigma_{z y}=\left(\alpha \mu+R^{2}\right) \frac{\partial \phi}{\partial y}+R(\alpha-\mu) \frac{\partial \psi}{\partial y} \\
\sigma_{x z}=\sigma_{z x}=\left(\alpha \mu+R^{2}\right) \frac{\partial \phi}{\partial x}+R(\alpha-\mu) \frac{\partial \psi}{\partial x} \\
H_{z y}=R\left(\alpha+\left(K_{1}-K_{2}\right)\right) \frac{\partial \phi}{\partial y}+\left(\alpha\left(K_{1}-K_{2}\right)-R^{2}\right) \frac{\partial \psi}{\partial y} \\
H_{z x}=R_{3}\left(\alpha+\left(K_{1}-K_{2}\right)\right) \frac{\partial \phi}{\partial x}+\left(\alpha\left(K_{1}-K_{2}\right)-R^{2}\right) \frac{\partial \psi}{\partial x}
\end{array}\right.
$$

The above formula can be regarded as anti-plane elasticity of one-dimensional quasicrystals and cubic quasicrystals as well. The difference lies in that $\mu, K_{1}-K_{2}$ and $R$ are replaced by $C_{44}, K_{44}$ and $R_{44}$ for cubic quasicrystals or replaced by $C_{44}, K_{2}$ and $R_{3}$ for one-dimensional hexagonal quasicrystals.

The solution of (7) can be determined by using the method for solving wave equations in mathematical physics.

\subsection{Example 2: Elasto-Dynamics of Hexagonal Quasicrystals}

In this case the generalized Hooke's law is [23]

$$
\left\{\begin{array}{l}
\sigma_{x x}=C_{11} \frac{\partial u_{x}}{\partial x}+C_{12} \frac{\partial u_{y}}{\partial y} \\
\sigma_{y y}=C_{12} \frac{\partial u_{x}}{\partial x}+C_{11} \frac{\partial u_{y}}{\partial y} \\
\sigma_{x y}=\sigma_{y x}=C_{66}\left(\frac{\partial u_{x}}{\partial y}+\frac{\partial u_{y}}{\partial x}\right) \\
\sigma_{z y}=\sigma_{y z}=C_{44} \frac{\partial u_{z}}{\partial y}+R_{3} \frac{\partial w_{z}}{\partial y} \\
\sigma_{x z}=\sigma_{z x}=C_{44} \frac{\partial u_{z}}{\partial x}+R_{3} \frac{\partial w_{z}}{\partial x} \\
H_{z y}=K_{2} \frac{\partial w_{z}}{\partial y}+R_{3} \frac{\partial u_{z}}{\partial y} \\
H_{z x}=K_{2} \frac{\partial w_{z}}{\partial x}+R_{3} \frac{\partial u_{z}}{\partial x}
\end{array}\right.
$$

The resulting wave equations are

$$
\begin{aligned}
& \nabla^{2} F=\frac{1}{c_{1}^{2}} \frac{\partial^{2} F}{\partial t^{2}}, \nabla^{2} G=\frac{1}{c_{2}^{2}} \frac{\partial^{2} G}{\partial t^{2}} \\
& \nabla^{2} \phi=\frac{1}{s_{1}^{2}} \frac{\partial^{2} \phi}{\partial t^{2}}, \nabla^{2} \psi=\frac{1}{s_{2}^{2}} \frac{\partial^{2} \psi}{\partial t^{2}}
\end{aligned}
$$

where

$$
\begin{gathered}
u_{x}=\frac{\partial F}{\partial x}+\frac{\partial G}{\partial y}, u_{y}=\frac{\partial F}{\partial y}-\frac{\partial G}{\partial x} \\
u_{z}=\alpha \phi-R \psi, w_{z}=R_{3} \phi+\alpha \psi \\
\alpha=\frac{1}{2}\left[C_{44}-K_{2}+\sqrt{\left(C_{44}-K_{2}\right)^{2}+4 R_{3}^{2}}\right] \\
c_{1}=\sqrt{C_{11} / \rho}, c_{2}=\sqrt{\left(C_{11}-C_{12}\right) / \rho} \\
s_{j}=\sqrt{\frac{\varepsilon_{j}}{\rho}}, j=1,2 \\
\varepsilon_{1,2}=\frac{1}{2}\left[C_{44}+K_{2} \pm \sqrt{\left(C_{44}-K_{2}\right)^{2}+4 R_{3}^{2}}\right]
\end{gathered}
$$




\subsection{Example 3: Elasto-Dynamics of Plane Elasticity of Dodecagonal Quasicrystals}

Let us take the $z$-axis as periodic direction in point group $12 \mathrm{~mm}$ dodecagonal quasicrystals. Suppose that the field variables not depends on the coordinate $z$, then the elasticity problem of dodecagonal quasicrystals can be turned into the plane elasticity problem and anti-plane elasticity problem. Meanwhile, here we only consider the plane elasticity, and the field quantities are

$$
\left\{\begin{array}{l}
u_{i}=u_{i}(x, y, t)(i=1,2) \\
w_{i}=w_{i}(x, y, t)(i=1,2)
\end{array}\right.
$$

in which $t$ represents time.

For the dodecagonal quasicrystals the generalized Hooke's law is as follows

$$
\left\{\begin{array}{l}
\sigma_{x x}=C_{12}\left(\varepsilon_{x x}+\varepsilon_{y y}\right)+\left(C_{11}-C_{12}\right) \varepsilon_{x x} \\
\sigma_{y y}=C_{12}\left(\varepsilon_{x x}+\varepsilon_{y y}\right)+\left(C_{11}-C_{12}\right) \varepsilon_{y y} \\
\sigma_{x y}=\sigma_{y x}=\left(C_{11}-C_{12}\right) \varepsilon_{x y} \\
H_{x x}=K_{1} w_{x x}+K_{2} w_{y y} \\
H_{y y}=K_{1} w_{y y}+K_{2} w_{x x} \\
H_{x y}=\left(K_{1}+K_{2}+K_{3}\right) w_{x y}+K_{3} w_{y x} \\
H_{y x}=\left(K_{1}+K_{2}+K_{3}\right) w_{y x}+K_{3} w_{x y}
\end{array}\right.
$$

In addition, we can see the phonon and phason are decoupled, i.e., $R_{i j k l}=R_{k l i j}=0$.

If we follow the standpoint of Bak [12,13], the related equilibrium equations with absence of the body force are

$$
\left\{\begin{array}{l}
\frac{\partial \sigma_{x x}}{\partial x}+\frac{\partial \sigma_{x y}}{\partial y}=\rho \frac{\partial^{2} u_{x}}{\partial t^{2}} \\
\frac{\partial \sigma_{y x}}{\partial x}+\frac{\partial \sigma_{y y}}{\partial y}=\rho \frac{\partial^{2} u_{y}}{\partial t^{2}} \\
\frac{\partial \sigma_{z x}}{\partial x}+\frac{\partial \sigma_{z y}}{\partial y}=\rho \frac{\partial^{2} u_{z}}{\partial t^{2}} \\
\frac{\partial H_{x x}}{\partial x}+\frac{\partial H_{x y}}{\partial y}=\rho \frac{\partial^{2} w_{x}}{\partial t^{2}} \\
\frac{\partial H_{y x}}{\partial x}+\frac{\partial H_{y y}}{\partial y}=\rho \frac{\partial^{2} w_{y}}{\partial t^{2}}
\end{array}\right.
$$

in which $\rho$ indicates the mass density of the materials.

Let us bring in the four displacement potential functions into the fixed coordinate system $\left(x_{1}, y, t\right)$ such that

$$
\left\{\begin{array}{l}
u_{x_{1}}=\frac{\partial F}{\partial x_{1}}+\frac{\partial G}{\partial y}, \quad u_{y}=\frac{\partial F}{\partial y}-\frac{\partial G}{\partial x_{1}} \\
w_{x_{1}}=\frac{\partial P}{\partial x_{1}}+\frac{\partial Q}{\partial y}, \quad w_{y}=-\frac{\partial P}{\partial y}+\frac{\partial Q}{\partial x_{1}}
\end{array}\right.
$$

Then the above basic equations are ascribed to the following wave Equations

$$
\nabla^{2} F=\frac{1}{c_{1}^{2}} \frac{\partial^{2} F}{\partial t^{2}}, \nabla^{2} G=\frac{1}{c_{2}^{2}} \frac{\partial^{2} G}{\partial t^{2}}, \nabla^{2} u_{z}=\frac{1}{c_{3}^{2}} \frac{\partial^{2} u_{z}}{\partial t^{2}}, \nabla^{2} P=\frac{1}{d_{1}^{2}} \frac{\partial^{2} P}{\partial t^{2}}, \nabla^{2} Q=\frac{1}{d_{2}^{2}} \frac{\partial^{2} Q}{\partial t^{2}}
$$

in which $\nabla^{2}=\partial^{2} / \partial x_{1}{ }^{2}+\partial^{2} / \partial y^{2}$ represents the two-dimensional Laplace operator, and

$$
c_{1}=\sqrt{\frac{C_{11}}{\rho}}, c_{2}=\sqrt{\frac{\left(C_{11}-C_{12}\right)}{2 \rho}}, d_{1}=\sqrt{\frac{K_{1}}{\rho}}, d_{2}=\sqrt{\frac{\left(K_{1}+K_{2}+K_{3}\right)}{\rho}}
$$

indicates the elastic wave speeds of the phonon field and phason field in the quasicrystal.

Equation (18) provide a macro-description of vibration and wave propagation in two-dimensional quasicrystals with 12-fold symmetry, which can be turned into the classical elastic wave equations. 
2.4. Example 4: The Approximate Form of Elasto-Dynamics of Two-Dimensional Elasticity (or Simplified Three-Dimensional Elasticity) of Icosahedral Quasicrystals

Considering the weak coupling case (the phonon-phason coupling is only in direction $z$ ), the final wave equations are

$$
\begin{gathered}
\nabla^{2} F=\frac{1}{c_{1}^{2}} \frac{\partial^{2} F}{\partial t^{2}}, \nabla^{2} G=\frac{1}{c_{2}^{2}} \frac{\partial^{2} G}{\partial t^{2}} \\
\nabla^{2} w_{x}=\frac{1}{c_{3}^{2}} \frac{\partial^{2} w_{x}}{\partial t^{2}}, \nabla^{2} w_{y}=\frac{1}{c_{4}^{2}} \frac{\partial^{2} w_{y}}{\partial t^{2}} \\
\nabla^{2} \phi=\frac{1}{d_{1}^{2}} \frac{\partial^{2} \phi}{\partial t^{2}}, \nabla^{2} \psi=\frac{1}{d_{2}^{2}} \frac{\partial^{2} \psi}{\partial t^{2}}
\end{gathered}
$$

in which $c_{1}, c_{2}$ defined by (19), $c_{3}=c_{4}=\sqrt{K_{1} / \rho}$ and $d_{1}, d_{2}$ are the $s_{1}, s_{2}$ defined by (19).

It is unavailable to obtain standard wave equations for elasto-dynamics of quasicrystals so far from the above four cases.

\subsection{A Moving Screw Dislocation in Anti-Plane Elasticity of Some Quasicrystals}

Let us consider a straight screw dislocation line parallel to the quasi-periodic axis which moves along a periodic $x$ axis in the periodic plane. Suppose the dislocation moves with a constant velocity $V$. Meanwhile, suppose that the dislocation has the Burgers vector is $\left(0,0, b_{3}^{\|}, 0, b_{3}^{\perp}\right)$. The dislocation conditions can be repressed by

$$
\int_{\Gamma} d u_{z}=\mathrm{b}_{3}^{\|}, \int_{\Gamma} d w_{z}=\mathrm{b}_{3}^{\perp}
$$

where $\Gamma$ denotes the Burgers circuit surroundings the core of the moving dislocation.

Henceforth we use $\left(x_{1}, x_{2}, t\right)$ to denote the fixed coordinates and $(x, y)$ to denote the moving coordinates. In order to facilitate this, we introduce the Galilean transformation

$$
x=x_{1}-V t, y=x_{2}
$$

Then the known wave Equation (7) can be ascribed to the Laplace equations, (i.e., $\left.\left(\nabla^{2}-\frac{1}{s_{1}^{2}} \frac{\partial^{2}}{\partial t^{2}}\right) \rightarrow \nabla_{1}^{2},\left(\nabla^{2}-\frac{1}{s_{2}^{2}} \frac{\partial^{2}}{\partial t^{2}}\right) \rightarrow \nabla_{2}^{2}, \nabla^{2}=\frac{\partial^{2}}{\partial x_{1}^{2}}+\frac{\partial^{2}}{\partial x_{2}^{2}}\right)$

$$
\nabla_{1}^{2} \phi=0, \nabla_{2}^{2} \psi=0
$$

where

$$
\begin{gathered}
\nabla_{1}^{2}=\frac{\partial^{2}}{\partial x^{2}}+\frac{\partial^{2}}{\partial y_{1}^{2}}, \nabla_{1}^{2}=\frac{\partial^{2}}{\partial x^{2}}+\frac{\partial^{2}}{\partial y_{2}^{2}} \\
y_{j}=\beta_{j} y_{,} \beta_{j}=\sqrt{1-V^{2} / s_{j}^{2}}, j=1,2
\end{gathered}
$$

The solution of Equation (23) is

$$
\phi=\operatorname{Im} F_{1}\left(z_{1}\right), \psi=\operatorname{Im} F_{2}\left(z_{2}\right)
$$

where

$$
z_{j}=x+i y_{j}(i=\sqrt{-1})
$$

$F_{1}\left(z_{1}\right)$ and $F_{2}\left(z_{2}\right)$ are analytic functions of $z_{1}$ and $z_{2}$; "Im" indicates the imaginary part of a complex function.

The analytic functions are determined from the boundary conditions (21) as

$$
\phi\left(x, y_{1}\right)=\frac{A_{1}}{2 \pi} \arctan \frac{y_{1}}{x}, \psi\left(x, y_{1}\right)=\frac{A_{2}}{2 \pi} \arctan \frac{y_{2}}{x}
$$


where

$$
A_{1}=\frac{\alpha \mathrm{b}_{3}^{\|}+R \mathrm{~b}_{3}^{\perp}}{\alpha^{2}+R^{2}}, A_{2}=\frac{\alpha \mathrm{b}_{3}^{\|}-R \mathrm{~b}_{3}^{\perp}}{\alpha^{2}+R^{2}}
$$

The displacement field in terms of the fixed coordinate is

$$
\begin{aligned}
& u_{z}(x, y, t)=\frac{1}{2 \pi\left(\alpha^{2}+R^{2}\right)}\left[\left(\alpha^{2} \arctan \frac{\beta_{1} y}{x-V t}+R^{2} \arctan \frac{\beta_{2} y}{x-V t}\right) \mathbf{b}_{3}^{\|}+\left(\arctan \frac{\beta_{1} y}{x-V t}-\arctan \frac{\beta_{2} y}{x-V t}\right) \alpha R b_{3}^{\perp}\right] \\
& w_{z}(x, y, t)=\frac{1}{2 \pi\left(\alpha^{2}+R^{2}\right)}\left[\left(R^{2} \arctan \frac{\beta_{1} y}{x-V t}+\alpha_{3}^{2} \arctan \frac{\beta_{2} y}{x-V t}\right) \mathbf{b}_{3}^{\perp}+\left(\arctan \frac{\beta_{1} y}{x-V t}-\arctan \frac{\beta_{2} y}{x-V t}\right) \alpha R \mathbf{b}_{3}^{\|}\right]
\end{aligned}
$$

If we use $W$ per unit length to denote the energy of moving dislocation, which is defined by the following integrals and composed of the potential energy $W_{p}$ and kinetic energy $W_{k}$

$$
W_{k}=\frac{1}{2} \rho \iint_{\Omega}\left[\left(\frac{\partial u_{z}}{\partial t}\right)^{2}+\left(\frac{\partial w_{z}}{\partial t}\right)^{2}\right] d x_{1} d x_{2}, W_{p}=\frac{1}{2} \iint_{\Omega}\left[\sigma_{i j} \frac{\partial u_{z}}{\partial t}+H_{i j} \frac{\partial w_{z}}{\partial t}\right] d x_{1} d x_{2}
$$

in which the integral around a ring $r_{0}<r<R_{0}, r_{0}$ indicates the size of the dislocation core and $R_{0}$ indicates the size of the dislocation net. In a general way $r_{0} \sim 10^{-8} \mathrm{~cm}$, and $R_{0} \sim 10^{4} r_{0}$. Substituting the stress and displacement expressions into (30), one obtains

$$
W_{k}=\frac{k_{k}}{4 \pi} \ln \frac{R_{0}}{r_{0}}, W_{p}=\frac{k_{p}}{4 \pi} \ln \frac{R_{0}}{r_{0}}
$$

where

$$
\begin{gathered}
k_{k}=\frac{\rho V^{2}\left(\alpha^{2}+R^{2}\right)}{2}\left(\frac{A_{1}^{2}}{\beta_{1}}+\frac{A_{2}^{2}}{\beta_{2}}\right) \\
k_{p}=\frac{A_{1}^{2}}{2}\left(\mu \alpha^{2}+\left(K_{1}-K_{2}\right) R^{2}+2 \alpha R^{2}\right)\left(\beta_{1}+\frac{1}{\beta_{1}}\right)+\frac{A_{2}^{2}}{2}\left(\mu R^{2}+\left(K_{1}-K_{2}\right) \alpha^{2}-2 \alpha R^{2}\right)\left(\beta_{2}+\frac{1}{\beta_{2}}\right)
\end{gathered}
$$

$A_{1}$ and $A_{2}$ are given by (28). The total energy is

$$
W=\frac{k_{k}+k_{p}}{4 \pi} \ln \frac{R_{0}}{r_{0}}
$$

When $V \rightarrow s_{2}$, i.e., $\beta_{2} \rightarrow 0$, the energy takes a limit to infinity; this is meaningless. Thus $s_{2}$ can be regarded as the limit of the velocity from a moving dislocation. When $V \ll s_{2}$, the energy $W$ can be expressed as

$$
W \approx W_{0}+\frac{1}{2} \rho V^{2}\left[\left(\mathbf{b}_{3}^{\|}\right)^{2}+\left(\mathbf{b}_{3}^{\perp}\right)^{2}\right] \frac{1}{4 \pi} \ln \frac{R_{0}}{r_{0}}=W_{0}+\frac{1}{2} m_{0} V^{2}
$$

where $W_{0}$ denotes the energy per unit length of a rest screw dislocation,

$$
W_{0}=\left[\mu\left(\mathrm{b}_{3}^{\| \mid}\right)^{2}+R\left(\mathrm{~b}_{3}^{\perp}\right)^{2}+2 \mathrm{~b}_{3}^{\|} \mathrm{b}_{3}^{\perp} R\right] \frac{1}{4 \pi} \ln \frac{R_{0}}{r_{0}}
$$

And $m_{0}$ is named by apparent mass of the dislocation per unit length:

$$
m_{0}=\left[\mu\left(\mathrm{b}_{3}^{\|}\right)^{2}+R\left(\mathrm{~b}_{3}^{\perp}\right)^{2}+2 \mathrm{~b}_{3}^{\|} \mathrm{b}_{3}^{\perp} R\right] \frac{1}{4 \pi} \ln \frac{R_{0}}{r_{0}}
$$

Let $V=0$, the solution is clear enough to be able to reduce to the solution of a static dislocation. Furthermore assume $b_{3}^{\perp}=0$ and $R=0$, we obtain the solution of reduced problem

$$
\begin{aligned}
& u_{z}(x-V t, y)=\frac{b}{2 \pi} \arctan \frac{\beta_{1} y}{x-V t} \\
& \sigma_{y z}=\sigma_{z y}=\frac{b}{2 \pi} \frac{\mu \beta_{1}(x-V t)}{(x-V t)^{2}+\beta_{1}^{2} y^{2}}
\end{aligned}
$$




$$
\begin{gathered}
\sigma_{x z}=\sigma_{z x}=-\frac{b}{2 \pi} \frac{\mu \beta_{1} y}{(x-V t)^{2}+\beta_{1}^{2} y^{2}} \\
W \approx\left(\mu b^{2}+\frac{1}{2} \rho V^{2} b^{2}\right) \frac{1}{4 \pi} \ln \frac{R_{0}}{r_{0}} \\
m_{0}=\frac{\rho b^{2}}{4 \pi} \ln \frac{R_{0}}{r_{0}}
\end{gathered}
$$

which is equal to the Eshellby solution for crystals.

The above solution can be reduced to the solutions anti-plane elasticity cases of cubic or hexagonal quasicrystals. The material constants should be replaced similarly with Section 2.1.

\subsection{A Mode III Moving Griffith Crack in Anti-Plane Elasticity}

Consider a moving Griffith crack of Mode III, which moves with constant speed $V$ along $x_{1}$ (see Figure 1). The fixed coordinates are $\left(x_{1}, x_{2}, t\right)$ and the moving coordinates are $(x, y)$.

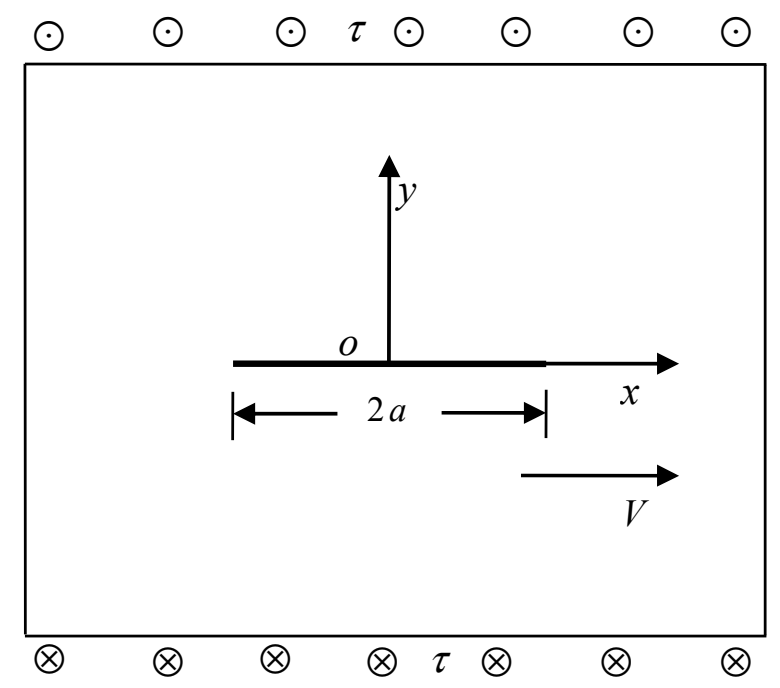

Figure 1. Moving Griffith crack of Mode III.

In the moving coordinates the boundary conditions are

$$
\begin{gathered}
\sqrt{x^{2}+y^{2}} \rightarrow \infty: \quad \sigma_{i j}=0, H_{i j}=0 \\
y=0,|x|<a: \quad \sigma_{y z}=-\tau, H_{y z}=0
\end{gathered}
$$

The general solution to the Laplace equations is

$$
\phi\left(x_{1}, y_{1}\right)=\operatorname{Re} F_{1}\left(z_{1}\right), \psi\left(x_{1}, y_{2}\right)=\operatorname{Re} F_{2}\left(z_{2}\right)
$$

where $F_{1}\left(z_{1}\right)$ and $F_{2}\left(z_{2}\right)$ are analytic functions of $z_{1}$ and $z_{2}$.

The problem is solved by using the conformal mapping

$$
z_{1}, z_{2}=\omega(\zeta)=\frac{a}{2}\left(\zeta+\zeta^{-1}\right)
$$

After some manipulations, we obtain the solution

$$
F_{1}\left(z_{1}\right)=F_{1}[\omega(\zeta)]=G_{1}(\zeta)=\frac{i \Delta_{1}}{\Delta} \zeta, F_{2}\left(z_{2}\right)=F_{2}[\omega(\zeta)]=G_{2}(\zeta)=\frac{i \Delta_{2}}{\Delta} \zeta
$$


in which

$$
\begin{gathered}
\Delta=\beta_{1} \beta_{2}\left[\left(\alpha \mu+R^{2}\right)\left(\alpha\left(K_{1}-K_{2}\right)-R^{2}\right)-R^{2}\left(\alpha+\left(K_{1}-K_{2}\right)\right)(\alpha-\mu)\right] \\
\Delta_{1}=\tau \alpha \beta_{2}\left(\alpha\left(K_{1}-K_{2}\right)-R^{2}\right) \\
\Delta_{2}=\tau \alpha \beta_{1} R\left(\alpha+\left(K_{1}-K_{2}\right)\right)
\end{gathered}
$$

The inverse mapping as

$$
\zeta=\omega^{-1}\left(z_{1}\right)=\frac{z_{1}}{a}-\sqrt{\left(\frac{z_{1}}{a}\right)^{2}-1}=\omega^{-1}\left(z_{2}\right)=\frac{z_{2}}{a}-\sqrt{\left(\frac{z_{2}}{a}\right)^{2}-1}
$$

The corresponding stresses are

$$
\begin{gathered}
\sigma_{y z}=\sigma_{z y}=\left(\alpha \mu+R^{2}\right) \beta_{1} \frac{\partial}{\partial y_{1}} \operatorname{Re} F_{1}\left(z_{1}\right)+R(\alpha-\mu) \beta_{2} \frac{\partial}{\partial y_{2}} \operatorname{Re} F_{2}\left(z_{2}\right) \\
\sigma_{x z}=\sigma_{z x}=\left(\alpha \mu+R^{2}\right) \frac{\partial}{\partial x} \operatorname{Re} F_{1}\left(z_{1}\right)+R_{3}(\alpha-\mu) \beta_{2} \frac{\partial}{\partial x} \operatorname{Re} F_{2}\left(z_{2}\right) \\
H_{z y}=R_{3}\left(\alpha+\left(K_{1}-K_{2}\right)\right) \beta_{1} \frac{\partial}{\partial y_{1}} \operatorname{Re} F_{1}\left(z_{1}\right)+\left(\alpha\left(K_{1}-K_{2}\right)-R^{2}\right) \frac{\partial}{\partial y_{2}} \operatorname{Re} F_{2}\left(z_{2}\right) \\
H_{z x}=R_{3}\left(\alpha+\left(K_{1}-K_{2}\right)\right) \beta_{1} \frac{\partial}{\partial x} \operatorname{Re} F_{1}\left(z_{1}\right)+\left(\alpha\left(K_{1}-K_{2}\right)-R^{2}\right) \frac{\partial}{\partial x} \operatorname{Re} F_{2}\left(z_{2}\right)
\end{gathered}
$$

Substituting (43) into (41) then into (44a), the stresses can be expressed in the explicit form as

$$
\begin{aligned}
\sigma_{y z}= & \sigma_{z y}=-\frac{\tau}{\Delta}\left(\alpha \mu+R^{2}\right) \beta_{1} \beta_{2}\left(\alpha\left(K_{1}-K_{2}\right)-R^{2}\right)\left[1-\frac{d}{\left(d_{1} d_{2}\right)^{\frac{1}{2}}} \cos \left(\theta-\frac{1}{2} \theta_{1}-\frac{1}{2} \theta_{2}\right)\right] \\
& +\frac{\tau}{\Delta} \beta_{1} \beta_{2} R^{2}\left(\alpha+\left(K_{1}-K_{2}\right)\right)(\alpha-\mu)\left[1-\frac{D}{\left(D_{1} D_{2}\right)^{\frac{1}{2}}} \cos \left(\theta-\frac{1}{2} \Theta_{1}-\frac{1}{2} \Theta_{2}\right)\right]
\end{aligned}
$$

with

$$
\begin{gathered}
d=\sqrt{x^{2}+y_{1}^{2}}, d_{1}=\sqrt{(x-a)^{2}+y_{1}^{2}}, d_{2}=\sqrt{(x+a)^{2}+y_{1}^{2}} \\
D=\sqrt{x^{2}+y_{2}^{2}}, D_{1}=\sqrt{(x-a)^{2}+y_{2}^{2}}, D_{2}=\sqrt{(x+a)^{2}+y_{2}^{2}} \\
\theta=\arctan \left(\frac{y_{1}}{x}\right), \theta_{1}=\arctan \left(\frac{y_{1}}{x-a}\right), \theta_{2}=\arctan \left(\frac{y_{1}}{x+a}\right) \\
\Theta=\arctan \left(\frac{y_{2}}{x}\right), \Theta_{1}=\arctan \left(\frac{y_{2}}{x-a}\right), \Theta_{2}=\arctan \left(\frac{y_{2}}{x+a}\right)
\end{gathered}
$$

It is easy to show that (45) satisfies the boundary conditions of the problem. Similarly, $\sigma_{x z}=\sigma_{z x}$, $H_{z x}$ and $H_{z y}$ can be expressed explicitly.

Let $y=0$ in Equation (45), we have

$$
\sigma_{y z}(x, 0)=\left\{\begin{array}{c}
\frac{x \tau}{\sqrt{x^{2}-a^{2}}}-\tau,|x|>a \\
-\tau,|x<a|
\end{array}\right.
$$

The stress is singular of order $(x-a)^{-1 / 2}$ when $x \rightarrow a$.

The stress intensity factor for Mode III for the phonon field is

$$
K_{I}^{\|}=\lim _{x \rightarrow a^{+}} \sqrt{\pi(x-a)} \sigma_{y z}(x, 0)=\sqrt{\pi a} \tau
$$

This is equal to the Yoffe solution [64], in which the stress intensity factor is also independent of the crack moving speed $V$. 
The energy of the moving crack is defined by

$$
\begin{gathered}
W=2 \int_{0}^{a}\left[\sigma_{z y}(x, 0) \oplus H_{z y}(x, 0)\right]\left[u_{z}(x, 0) \oplus w_{z}(x, 0)\right] d x \\
=\frac{1}{\Delta}\left(\Delta_{1} \alpha-\Delta_{2} R\right) \tau \pi a=\frac{1}{\Delta}\left[\alpha \beta_{2}\left(\alpha\left(K_{1}-K_{2}\right)-R^{2}\right)-\beta_{1} R^{2}\left(\alpha+\left(K_{1}-K_{2}\right)\right)\right] \pi a^{2} \tau
\end{gathered}
$$

The crack energy release rate is

$$
G=\frac{1}{2} \frac{\partial W}{\partial a}=\frac{1}{2 \Delta}\left[\alpha \beta_{2}\left(\alpha\left(K_{1}-K_{2}\right)-R^{2}\right)-\beta_{1} R^{2}\left(\alpha+\left(K_{1}-K_{2}\right)\right]\left(K_{I}^{\|}\right)^{2}\right.
$$

The above results hold for anti-plane elasticity of three-dimensional cubic and one-dimensional hexagonal quasicrystals as well provided that the material constants $\mu, K_{1}-K_{2}$ and $R$ are replaced by $C_{44}, K_{44}$ and $R_{44}$ or by $C_{44}, K_{2}$ and $R_{3}$, respectively.

\subsection{The Moving Dugdale Model for Plane Elasticity of Dodecagonal Quasicrystals}

Fan and his co-workers finished many systematical studies on elasticity and elasto-dynamics of quasicrystals. In the following sections, we will reveal the general solutions of the dynamic problems of the point group $12 \mathrm{~mm}$ two-dimensional dodecagonal quasicrystals and give an example using this approach. A moving Dugdale model of the point group $12 \mathrm{~mm}$ two-dimensional dodecagonal quasicrystals is solved in this paper. The Dugdale-type plastic zones ahead of the crack tip are also obtained for when a crack in a dodecagonal quasicrystal is moving or growing at a constant speed $V$ in the direction $O x_{1}$. We introduce the Galileo transformation for convenience

$$
x=x_{1}-V t, y=y
$$

Then the wave equations are reduced to the Laplace equations in the moving coordinate system $(x, y)$

$$
\left\{\begin{array}{l}
\left(\frac{\partial^{2}}{\partial x^{2}}+\frac{\partial^{2}}{\partial y_{1}^{2}}\right) F\left(x, y_{1}\right)=0,\left(\frac{\partial^{2}}{\partial x^{2}}+\frac{\partial^{2}}{\partial y_{2}^{2}}\right) G\left(x, y_{2}\right)=0 \\
\left(\frac{\partial^{2}}{\partial x^{2}}+\frac{\partial^{2}}{\partial y_{3}^{2}}\right) P\left(x, y_{3}\right)=0,\left(\frac{\partial^{2}}{\partial x^{2}}+\frac{\partial^{2}}{\partial y_{4}^{2}}\right) Q\left(x, y_{4}\right)=0
\end{array}\right.
$$

where

$$
\left\{\begin{array}{c}
y_{1}=\alpha_{1} y, y_{2}=\alpha_{2} y, y_{3}=\alpha_{3} y, y_{4}=\alpha_{4} y \\
\alpha_{1}=\left(1-V^{2} c_{1}^{2}\right)^{\frac{1}{2}}, \alpha_{2}=\left(1-V^{2} c_{2}^{2}\right)^{\frac{1}{2}}, \alpha_{3}=\left(1-V^{2} d_{1}^{2}\right)^{\frac{1}{2}}, \alpha_{4}=\left(1-V^{2} d_{2}^{2}\right)^{\frac{1}{2}}
\end{array}\right.
$$

We give a general solution to the Equation (52) by using complex variable function theory

$$
\left\{\begin{array}{l}
F\left(x, y_{1}\right)=F_{1}\left(z_{1}\right)+\overline{F_{1}\left(z_{1}\right)}, G\left(x, y_{2}\right)=i\left[F_{2}\left(z_{2}\right)-\overline{F_{2}\left(z_{2}\right)}\right] \\
P\left(x, y_{3}\right)=F_{3}\left(z_{3}\right)+\overline{F_{3}\left(z_{3}\right)}, Q\left(x, y_{4}\right)=i\left[F_{4}\left(z_{4}\right)-\overline{F_{4}\left(z_{4}\right)}\right]
\end{array}\right.
$$

where $F_{i}\left(z_{i}\right)(i=1,2,3,4)$ are four analytic functions of the complex variables $z_{i}(i=1,2,3,4)$ given by

$$
z_{1}=x+i y_{1}, z_{2}=x+i y_{2}, z_{3}=x+i y_{3}, z_{4}=x+i y_{4}
$$

and $\overline{F_{i}\left(z_{i}\right)}$ are the complex conjugations of $F_{i}\left(z_{i}\right)$.

It is noted that some new symbols for convenience

$$
\left\{\begin{array}{l}
\Phi\left(z_{1}\right)=\frac{d F_{1}\left(z_{1}\right)}{d z_{1}}=F_{1}^{\prime}\left(z_{1}\right), \Psi\left(z_{2}\right)=\frac{d F_{2}\left(z_{2}\right)}{d z_{2}}=F_{2}^{\prime}\left(z_{2}\right) \\
\Phi_{1}\left(z_{3}\right)=\frac{d F_{3}\left(z_{3}\right)}{d z_{3}}=F_{3}^{\prime}\left(z_{3}\right), \Psi_{1}\left(z_{4}\right)=\frac{d F_{4}\left(z_{4}\right)}{d z_{4}}=F_{4}^{\prime}\left(z_{4}\right)
\end{array}\right.
$$


and we will obtain the complex expressions of the displacements

$$
\left\{\begin{array}{c}
u_{x}=2 \operatorname{Re}\left[\Phi\left(z_{1}\right)-\alpha_{2} \Psi\left(z_{2}\right)\right] \\
u_{y}=-2 \operatorname{Im}\left[\alpha_{1} \Phi\left(z_{1}\right)-\Psi\left(z_{2}\right)\right] \\
w_{x}=2 \operatorname{Re}\left[\Phi_{1}\left(z_{3}\right)-\alpha_{4} \Psi_{1}\left(z_{4}\right)\right] \\
w_{y}=-2 \operatorname{Im}\left[\alpha_{3} \Phi_{1}\left(z_{3}\right)-\Psi_{1}\left(z_{4}\right)\right]
\end{array}\right.
$$

and the stresses expressed by

$$
\left\{\begin{array}{l}
\sigma_{x x}+\sigma_{y y}=2 \mu\left(\alpha_{1}^{2}-\alpha_{2}^{2}\right)\left[\Phi^{\prime}\left(z_{1}\right)+\overline{\Phi^{\prime}\left(z_{1}\right)}\right] \\
\sigma_{x x}-\sigma_{y y}+i 2 \sigma_{x y}=2 \mu\left[\begin{array}{l}
\left(1-\alpha_{1}\right)^{2} \Phi^{\prime}\left(z_{1}\right)+\left(1+\alpha_{1}\right)^{2} \overline{\Phi^{\prime}\left(z_{1}\right)}+ \\
\left(1-\alpha_{2}\right)^{2} \Psi^{\prime}\left(z_{2}\right)+\left(1+\alpha_{2}\right)^{2} \overline{\Psi^{\prime}\left(z_{2}\right)}
\end{array}\right] \\
H_{x x}+H_{y y}=2\left(K_{2}+K_{3}\right)\left(\alpha_{3}^{2}-\alpha_{4}^{2}\right)\left[\Phi_{1}^{\prime}\left(z_{3}\right)+\overline{\Phi_{1}^{\prime}\left(z_{3}\right)}\right] \\
H_{x x}-H_{y y}+i 2 H_{x y}=2\left(K_{2}+K_{3}\right)\left[\begin{array}{l}
\left(1-\alpha_{3}\right)^{2} \Phi_{1}^{\prime}\left(z_{3}\right)+\left(1+\alpha_{3}\right)^{2} \overline{\Phi_{1}^{\prime}\left(z_{3}\right)}+ \\
\left(1-\alpha_{4}\right)^{2} \Psi_{1}^{\prime}\left(z_{4}\right)+\left(1+\alpha_{4}\right)^{2} \overline{\Psi_{1}^{\prime}\left(z_{4}\right)}
\end{array}\right]
\end{array}\right.
$$

Suppose the phason field has no contribution on phonon field deformation, we will only discuss the phonon field, which can provide a macroscopic description for this kind dynamic problem. To proceed we represent $\Phi\left(z_{1}\right)$ and $\Psi\left(z_{2}\right)$ in series expansion form which are in agreement with the boundary conditions

$$
\left\{\begin{array}{l}
\Phi\left(z_{1}\right)=\frac{\sigma_{x x}^{(\infty)}+\sigma_{y y}^{(\infty)}}{4 \mu\left(\alpha_{1}^{2}+\alpha_{2}^{2}\right)}-i \frac{\sigma_{x y}^{(\infty)}}{4 \mu \alpha_{1}^{2}}+\sum_{n=1}^{\infty} z_{1}^{-n} \\
\Psi\left(z_{2}\right)=\frac{\sigma_{y y}^{(\infty)}}{4 \mu \alpha_{2}}+\frac{\left(1+\alpha_{2}^{2}\right)\left(\sigma_{x x}^{(\infty)}+\sigma_{y y}^{(\infty)}\right)}{8 \mu \alpha_{2}\left(\alpha_{1}^{2}-\alpha_{2}^{2}\right)}+\sum_{n=1}^{\infty} z_{2}^{-n}
\end{array}\right.
$$

and $\sigma_{i j}^{(\infty)}$ denotes the stresses at infinity.

With the help of a conformal transformation

$$
z_{1}, z_{2}=\omega(\zeta)
$$

The exterior of $\Gamma$ in the $z_{1}$-plane or $z_{2}$-plane is mapped into the interior of the unit circle $\gamma$ in the $\zeta$-plane. Supposing the distribution of normal and shear stresses on $\Gamma$ to be $-\sigma_{s} q_{1}(x)$ and $-\tau_{s} q_{2}(x)$, from the relations in the Equation (58) we have

$$
\left\{\begin{array}{l}
\operatorname{Re}\left[\left(1+\alpha_{2}^{2}\right) \Phi^{\prime}\left(z_{1}\right)-2 \alpha_{2} \Psi^{\prime}\left(z_{2}\right)\right]=\frac{\sigma_{s}}{2 \mu} q_{1}(x) \\
\operatorname{Im}\left[2 \alpha_{1} \Phi^{\prime}\left(z_{1}\right)-\left(1+\alpha_{2}^{2}\right) \Psi^{\prime}\left(z_{2}\right)\right]=\frac{\tau_{s}}{2 \mu} q_{2}(x)
\end{array} \quad(x, y \in \Gamma)\right.
$$

where $\sigma_{s}$ and $\tau_{s}$ are material constants and $q_{1}(x)$ and $q_{2}(x)$ are known functions.

We begin by writing unknown functions in terms of the Equation (60)

$$
\Phi\left(z_{1}\right)=\Phi[\omega(\zeta)]=\Phi_{*}(\zeta), \Psi\left(z_{2}\right)=\Psi[\omega(\zeta)]=\Psi_{*}(\zeta)
$$

and

$$
\Phi^{\prime}\left(z_{1}\right)=\Phi_{*}^{\prime}(\zeta) / \omega^{\prime}(\zeta), \Psi^{\prime}\left(z_{2}\right)=\Psi_{*}^{\prime}(\zeta) / \omega^{\prime}(\zeta)
$$

Then the boundary conditions (61) may be rewritten as

$$
\left\{\begin{array}{l}
\frac{1}{2 \pi i} \int_{\gamma} \frac{G_{1}(\sigma) d \sigma}{\sigma-\zeta}+\frac{1}{2 \pi i} \int_{\gamma} \frac{\omega^{\prime}(\sigma)}{\overline{\omega^{\prime}(\sigma)}} \frac{\overline{G_{1}(\sigma)} d \sigma}{\sigma-\zeta}=\frac{\sigma_{s}}{2 \mu} \cdot \frac{1}{2 \pi i} \int_{\gamma} \frac{\omega^{\prime}(\sigma) q_{1}^{*}(\sigma) d \sigma}{\sigma-\zeta} \\
\frac{1}{2 \pi i} \int_{\gamma} \frac{G_{2}(\sigma) d \sigma}{\sigma-\zeta}-\frac{1}{2 \pi i} \int_{\gamma} \frac{\omega^{\prime}(\sigma)}{\overline{\omega^{\prime}(\sigma)}} \frac{\overline{G_{2}(\sigma)} d \sigma}{\sigma-\zeta}=\frac{\tau_{s}}{2 \mu} \cdot \frac{1}{2 \pi i} \int_{\gamma} \frac{\omega^{\prime}(\sigma) q_{2}^{*}(\sigma) d \sigma}{\sigma-\zeta}
\end{array}\right.
$$


where $|\zeta|<1$ and

$$
\left\{\begin{array}{l}
G_{1}(\sigma)=\left(1+\alpha_{2}^{2}\right) \Phi_{*}^{\prime}(\sigma)-2 \alpha_{2} \Psi_{*}^{\prime}(\sigma) \\
G_{2}(\sigma)=2 \alpha_{1} \Phi_{*}^{\prime}(\sigma)-\left(1+\alpha_{2}^{2}\right) \Psi_{*}^{\prime}(\sigma)
\end{array}\right.
$$

and

$$
\left\{\begin{array}{l}
q_{1}(x)=q_{1}[\omega(\sigma)]=q_{1}^{*}(\sigma) \\
q_{2}(x)=q_{2}[\omega(\sigma)]=q_{2}^{*}(\sigma)
\end{array}\right.
$$

Thus the dynamic crack problem is reduced to the boundary value problem $(64)$ of $G_{1}(\zeta)$ and $G_{2}(\zeta)$, which are analytic functions in the interior of the unit circle $\gamma$. For concrete functions $\omega(\zeta), q_{1}^{*}(\sigma)$ and $q_{2}^{*}(\sigma)$, the solutions can be obtained.

In general, the plastic deformation will occur in the vicinity of the crack tip when it expands or propagates. The zone is placed at the crack tip and subjected to so-called "cohesive forces" impeding the fracture growth. So we extend the Dugdale-Barenblatt model for the moving crack problem. As shown in Figure 2, the material is loaded to a pulling stress $\sigma_{y y}=\sigma^{(\infty)}$ at infinity, and the so-called coupled cohesive force zone is located at $y=0, l<|x|<l+b$, where $b$ is unknown, and in the cohesive force zone $\sigma_{y y}$ is equal to the stress $\sigma_{s}$ which represents the yield stress of the quasicrystals. Obviously, this simplified model is linearized. So we can utilize the superposition principle to prescribe the above problem apart from a negligible constant term as follows:

$$
\left\{\begin{array}{l}
y=0,|x|<l: \sigma_{y y}=-\sigma^{(\infty)}, H_{y y}=0, \sigma_{x y}=0, H_{x y}=0 \\
y=0, l<|x|<l+b: \sigma_{y y}=\sigma_{s}-\sigma^{(\infty)}, H_{y y}=0, \sigma_{x y}=0, H_{x y}=0 \\
\left(x^{2}+y^{2}\right)^{\frac{1}{2}} \rightarrow \infty: \sigma_{i j}=0, H_{i j}=0
\end{array}\right.
$$

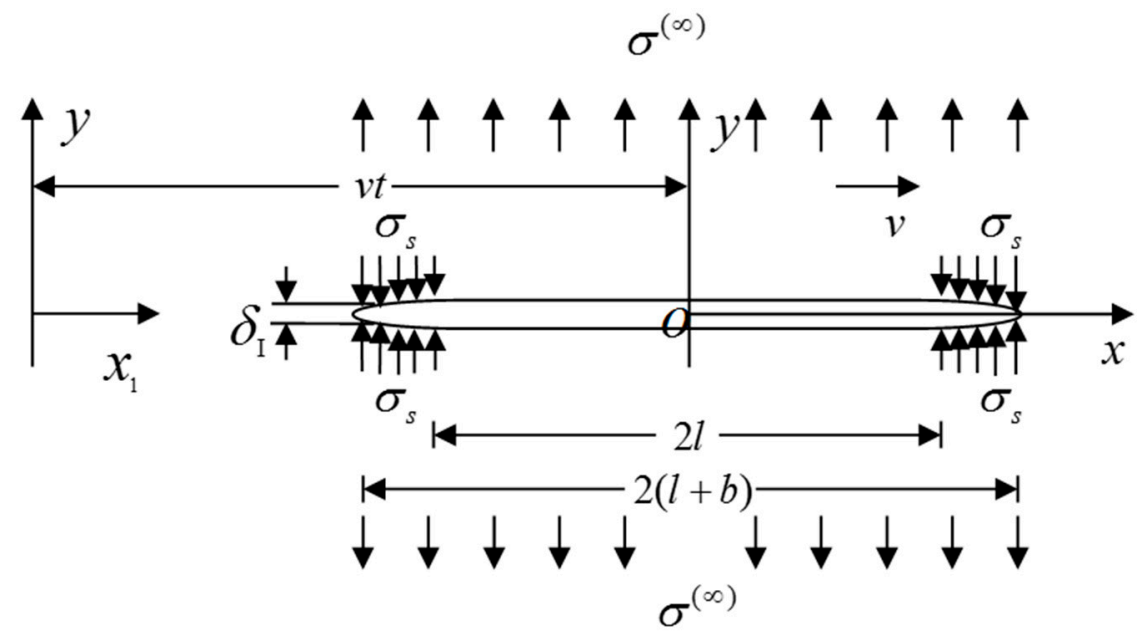

Figure 2. A moving Dugdale model for dynamic problem of dodecagonal quasicrystals.

For this problem the mapping function reads

$$
z_{1}, z_{2}=\frac{l+b}{2}\left(\zeta+\frac{1}{\zeta}\right)
$$

Substituting Equations (67) and (68) into (66), on the basis of the Cauchy integral theory and the analytic continuity principle we obtain

$$
\left\{\begin{array}{l}
\Phi^{\prime}\left(z_{1}\right)=\frac{1}{\mu} \frac{1+\alpha_{2}^{2}}{\left(1+\alpha_{2}^{2}\right)^{2}-4 \alpha_{1} \alpha_{2}}\left(\frac{2 \theta_{1}}{\pi \zeta^{2}} \sigma_{s}-\sigma^{(\infty)}\right) \frac{\zeta^{2}}{\zeta^{2}-1}+\frac{1}{2 \pi i} \frac{\sigma_{s}}{\mu} \frac{1+\alpha_{2}^{2}}{\left(1+\alpha_{2}^{2}\right)^{2}-4 \alpha_{1} \alpha_{2}} \ln \frac{e^{2 i \theta_{1}}-\zeta^{2}}{e^{-2 i \theta_{1}}-\zeta^{2}} \\
\Psi^{\prime}\left(z_{2}\right)=\frac{1}{\mu} \frac{2 \alpha_{1}}{\left(1+\alpha_{2}^{2}\right)^{2}-4 \alpha_{1} \alpha_{2}}\left(\frac{2 \theta_{1}}{\pi \zeta^{2}} \sigma_{s}-\sigma^{(\infty)}\right) \frac{\zeta^{2}}{\zeta^{2}-1}+\frac{1}{2 \pi i} \frac{\sigma_{s}}{\mu} \frac{2 \alpha_{1}}{\left(1+\alpha_{2}^{2}\right)^{2}-4 \alpha_{1} \alpha_{2}} \ln \frac{e^{2 i \theta_{1}}-\zeta^{2}}{e^{-2 i \theta_{1}}-\zeta^{2}}
\end{array}\right.
$$


where the angle $\theta_{1}$ located in the unit circle $\gamma$ in the $\zeta$-plane corresponds to the crack tip and can be determined by $\cos \theta_{1}=l /(l+b)$, and $\zeta= \pm 1$ corresponds to the ends of the cohesive force zone.

Because the stresses at the ends of the cohesive force zone, i.e., at $y=0, x= \pm(l+b)$ at the $z$-plane or in $\zeta= \pm 1$ at the $\zeta$-plane must be equal to a finite value, there is no stress singularity. From the known Equations (58) and (69), these conditions lead to

$$
\frac{1}{\mu} \frac{1+\alpha_{2}^{2}}{\left(1+\alpha_{2}^{2}\right)^{2}-4 \alpha_{1} \alpha_{2}}\left(\frac{2 \theta_{1}}{\pi} \sigma_{s}-\sigma^{(\infty)}\right)=0,
$$

and combining with the condition $\cos \theta_{1}=l /(l+b)$, we can easily obtain the size of the cohesive force zone

$$
b=l\left[\sec \left(\frac{\pi \sigma^{(\infty)}}{2 \sigma_{s}}\right)-1\right]
$$

This value is corresponding to the result of the Dugdale model in nonlinear static fracture of isotropic body.

Using the displacement formulas and the integration of the functions (70) and noting the Equation (71), we can get the displacement $u_{y}$

$$
\begin{gathered}
u_{y}=\frac{1}{\pi} \frac{(l+b) \sigma_{s}}{\mu} \frac{\alpha_{1}\left(\alpha_{2}^{2}-1\right)}{\left(1+\alpha_{2}^{2}\right)^{2}-4 \alpha_{1} \alpha_{2}}\left\{\operatorname{Im}\left(\frac{2 \theta_{1} \sigma_{s}}{\zeta}+\pi \sigma^{(\infty)} \zeta\right)\right. \\
\left.+\operatorname{Re}\left[\left(e^{i \theta}+\overline{e^{i \theta_{1}}}\right) \ln \frac{\zeta^{2}-e^{2 i \theta_{1}}}{\zeta^{2}-e^{-2 i \theta_{1}}}-\left(e^{i \theta_{1}}-\overline{e^{i \theta_{1}}}\right) \ln \frac{\left(\zeta+e^{i \theta_{1}}\right)\left(\zeta-e^{i \theta_{1}}\right)}{\left(\zeta+e^{i \theta_{1}}\right)\left(\zeta-e^{i \theta_{1}}\right)}\right]\right\}
\end{gathered}
$$

in which $\zeta$ take its value on the circle i.e., $\zeta=\sigma=e^{i \theta}$.

According to the following formula, we take the limitation and the dynamic crack tip opening displacement is found to be

$$
\delta_{\mathrm{I}}^{\text {dynamic }}=\lim _{\theta \rightarrow \theta_{1}} 2 u_{y}=f(v) \delta_{\mathrm{I}}^{\text {static }}
$$

in which

$$
\begin{gathered}
f(v)= \begin{cases}(1+v) \frac{\alpha_{1}\left(1-\alpha_{2}^{2}\right)}{4 \alpha_{1} \alpha_{2}-\left(1+\alpha_{2}^{2}\right)^{2}}, & \text { plane stress state } \\
\frac{\alpha_{1}\left(1-\alpha_{2}^{2}\right)}{4 \alpha_{1} \alpha_{2}-\left(1+\alpha_{2}^{2}\right)^{2}} /(1-v), & \text { plane strain state }\end{cases} \\
\delta_{\mathrm{I}}^{\text {static }}=\frac{8 l \sigma_{s}}{\pi E^{\prime}} \ln \sec \left(\frac{\pi \sigma^{(\infty)}}{2 \sigma_{s}}\right) \text { and } E^{\prime}= \begin{cases}E & \text { plane stress state } \\
E /\left(1-v^{2}\right) & \text { plane strain state }\end{cases}
\end{gathered}
$$

In these formulas the expression of $\delta_{\mathrm{I}}^{\text {static }}$ is equal to the result of statics, in which $E$ and $v$ are Young's modulus and Poission's ratio respectively.

\section{Elasto-/Hydro-Dynamics of Quasicrystals Based on the Argument of Lubensky et al.}

The formulation in the preceding sections come from Bak's standpoint, which is based on the equations of motion

$$
\rho \frac{\partial^{2} u_{i}}{\partial t^{2}}=\frac{\partial \sigma_{i j}}{\partial x_{j}}, \rho \frac{\partial^{2} w_{i}}{\partial t^{2}}=\frac{\partial H_{i j}}{\partial x_{j}}
$$

Fan et al. [23] discard some ideas of both the arguments of Bak and Lubensky and presented the following equations of motion under linear and small deformation to replace the wave equations

$$
\rho \frac{\partial^{2} u_{i}}{\partial t^{2}}=\frac{\partial \sigma_{i j}}{\partial x_{j}}, \kappa \frac{\partial w_{i}}{\partial t}=\frac{\partial H_{i j}}{\partial x_{j}}
$$


The first equation of (57) is the usual equation of elasto-dynamics, in which $\rho$ is the mass density; the other one is a diffusion equation, where $\kappa=1 / \Gamma_{w}$, in which $\Gamma_{w}$ the kinematics coefficient $\Gamma_{w}$ of the phason field introduced by Lubensky et al. [52]. It is obvious that the second equation of (57) is a linearized equation of hydrodynamics of quasicrystals of Lubensky et al. The dynamic equations can be regarded as the elasto-/hydro-dynamic equations of quasicrystals. This operation is believed to be reconcile the contradiction.

\subsection{Application 1 of Elasto-/Hydro-Dynamics-Approximate Solution for a Moving Screw Dislocation in} Anti-Plane Elasticity

Consider a moving dislocation in anti-plane problem of three-dimensional icosahedral or cubic or one-dimensional hexagonal quasicrystal. In this case the stress and strain relations are regarded as Equation (4). The basic difference lies in the equations of motion, they are

$$
\mu \nabla^{2} u_{z}+R \nabla^{2} w_{z}=\rho \frac{\partial^{2} u_{z}}{\partial t^{2}}, R \nabla^{2} u_{z}+\left(K_{1}-K_{2}\right) \nabla^{2} w_{z}=\kappa \frac{\partial w_{z}}{\partial t}
$$

Equation (78) are wave-diffusion equations.

Suppose the screw dislocation has a speed $V$, which moves along direction $O x$. So we can easily write the boundary conditions

$$
\begin{gathered}
\sqrt{x^{2}+y^{2}} \rightarrow \infty: \sigma_{i j}(x, y, t) \rightarrow 0, \quad H_{i j}(x, y, t) \rightarrow 0 \\
\int_{\Gamma} d u_{z}=b_{3}^{\|} \int_{\Gamma} d w_{z}=b_{3}^{\perp}
\end{gathered}
$$

The problem (78), (79) is solved by means of perturbation incorporating the varitional method. $R / \mu$ is a small quantity, which can serve as the perturbation parameter. First, we determine the zeroth-order solution. So the governing equation is

$$
\begin{aligned}
& \rho \frac{\partial^{2} u^{0}}{\partial t^{2}}-\mu \nabla^{2} u^{0}=0 \\
& \kappa \frac{\partial w^{0}}{\partial t}-\left(K_{1}-K_{2}\right) \nabla^{2} w^{0}=0
\end{aligned}
$$

where $u^{0}, w^{0}$ signify the zeroth-order solution.

Recall the Galileo transformation

$$
x=x_{1}-V t
$$

We can obtain

$$
\frac{\partial}{\partial t}=-V \frac{\partial}{\partial x}, \frac{\partial^{2}}{\partial t^{2}}=V^{2} \frac{\partial^{2}}{\partial x^{2}}
$$

Putting (82) into (80) yields

$$
\begin{aligned}
& \frac{\partial^{2} u^{0}}{\partial x^{2}}+\frac{1}{\beta_{1}^{2}} \frac{\partial^{2} u^{0}}{\partial y^{2}}=0 \\
& \frac{\partial^{2} w^{0}}{\partial x^{2}}+\beta_{2}^{2} \frac{\partial w^{0}}{\partial x}+\frac{\partial^{2} w^{0}}{\partial y^{2}}=0
\end{aligned}
$$

where

$$
\beta_{1}^{2}=1-\frac{V^{2}}{c^{2}}, \beta_{2}^{2}=\frac{V \kappa}{K_{1}-K_{2}}=\frac{V}{\left(K_{1}-K_{2}\right) \Gamma_{w}}
$$

in which $c=\sqrt{\mu / \rho}$ is the transverse wave velocity of the phonon field in an icosahedral quasicrystal.

Consider the dislocation conditions in (79), we have the following two sets of equations

$$
\left\{\begin{array}{l}
\frac{\partial^{2} u^{0}}{\partial x^{2}}+\frac{1}{\beta_{1}^{2}} \frac{\partial^{2} u^{0}}{\partial y^{2}}=0 \\
\int_{\Gamma} d u^{0}=b_{3}^{\|}
\end{array}\right.
$$




$$
\left\{\begin{array}{l}
\frac{\partial^{2} w^{0}}{\partial x^{2}}+\beta_{2}^{2} \frac{\partial w^{0}}{\partial x}+\frac{\partial^{2} w^{0}}{\partial y^{2}}=0 \\
\int_{\Gamma} d w^{0}=b_{3}^{\perp}
\end{array}\right.
$$

The solution is

$$
u^{0}=\frac{b_{3}^{\|}}{2 \pi} \arctan \frac{\beta_{1} y}{x}
$$

To solve Equation (85b), let

$$
w^{0}=w_{1}+w_{2}
$$

in which $w_{1}$ satisfies

$$
\left\{\begin{array}{l}
\frac{\partial^{2} w_{1}}{\partial x^{2}}+\frac{\partial^{2} w_{1}}{\partial y^{2}}=0 \\
\int_{\Gamma} d w_{1}=b_{3}^{\perp}
\end{array}\right.
$$

which has the solution

$$
w_{1}=\frac{b_{3}^{\perp}}{2 \pi} \arctan \frac{y}{x}
$$

and $w_{2}$ satisfies

$$
\left\{\begin{array}{l}
\frac{\partial^{2} w_{2}}{\partial x^{2}}+\beta_{2}^{2} \frac{\partial w_{2}}{\partial x}+\frac{\partial^{2} w_{2}}{\partial y^{2}}=\frac{\beta_{2} b_{3}^{\perp} y}{2 \pi\left(x^{2}+y^{2}\right)} \\
\int_{\Gamma} d w_{2}=0
\end{array}\right.
$$

The Equation in (90) is very complicated. It can be solved approximately by the variational method. Without giving the detail, the approximate solution is

$$
w_{2}=-\frac{b_{3}^{\perp}}{2 \pi} \frac{\beta_{2}^{2} x^{2} y}{\left(x^{2}+y^{2}\right)\left(1+\beta_{2}^{2} x\right)}
$$

From Equations (77), (79) and (91), we obtain

$$
w^{0}=\frac{b_{3}^{\perp}}{2 \pi}\left(\arctan \frac{y}{x}-\frac{\beta_{2}^{2} x^{2} y}{\left(x^{2}+y^{2}\right)\left(1+\beta_{2}^{2} x\right)}\right)
$$

The governing Equation (78) for the first-order solution are

$$
\begin{aligned}
& \rho \frac{\partial^{2} u^{1}}{\partial t^{2}}-\mu \nabla^{2} u^{1}=R \nabla^{2} w^{0} \\
& \kappa \frac{\partial w^{1}}{\partial t}-\left(K_{1}-K_{2}\right) \nabla^{2} w^{1}=R \nabla^{2} u^{0}
\end{aligned}
$$

where $u^{1}, w^{1}$ signify the first -order solution.

Solving the governing Equation (93) with the boundary condition (79), and using the variational method weobtain the first-order solution as follows

$$
\begin{aligned}
& u^{1}=-\frac{b \frac{1}{2}}{2 \pi} \frac{R}{\left(\rho V^{2}-\mu\right)} \frac{2 \beta_{2}^{2} x^{2} y\left(3 x^{2}-y^{2}+6 \beta_{2}^{2} x^{3}+2 \beta_{2}^{4} x^{4}\right)}{\left(x^{2}+y^{2}\right)^{2}\left(1+\beta_{2}^{2} x\right)^{3}} \\
& w^{1}=-\frac{b_{3}^{\|} R}{2 \pi\left(K_{1}-K_{2}\right)} \frac{2\left(\beta_{1}^{3}-\beta_{1}\right) x^{3} y}{\left(x^{2}+\beta_{1}^{2} y^{2}\right)^{2}\left(1+\beta_{2}^{2} x\right)}
\end{aligned}
$$


The displacements of the moving dislocation problem follows the above-mentioned perturbation solution

$$
\begin{aligned}
& u_{z}\left(x_{1}, y, t\right) \approx u_{z}^{0}\left(x_{1}, y, t\right)+u_{z}^{1}\left(x_{1}, y, t\right) \\
& =\frac{b_{3}^{\|}}{2 \pi} \arctan \frac{\beta_{1} y}{x}-\frac{b_{3}^{\perp}}{2 \pi} \frac{R}{\left(\rho V^{2}-\mu\right)} \frac{2 \beta_{2}^{2} x^{2} y\left(3 x^{2}-y^{2}+6 \beta_{2}^{2} x^{3}+2 \beta_{2}^{4} x^{4}\right)}{\left(x^{2}+y^{2}\right)^{2}\left(1+\beta_{2}^{2} x\right)^{3}} \\
& w_{z}\left(x_{1}, y, t\right) \approx w_{z}^{0}\left(x_{1}, y, t\right)+w_{z}^{1}\left(x_{1}, y, t\right) \\
& =\frac{b_{3}^{1}}{2 \pi}\left[\arctan \frac{y}{x}-\frac{\beta_{2}^{2} x^{2} y}{\left(1+\beta_{2}^{2} x\right)\left(x^{2}+y^{2}\right)}\right]-\frac{b_{3}^{\|} R}{2 \pi\left(K_{1}-K_{2}\right)} \frac{2\left(\beta_{1}^{3}-\beta_{1}\right) x^{3} y}{\left(x^{2}+\beta_{1}^{2} y^{2}\right)^{2}\left(1+\beta_{2}^{2} x\right)}
\end{aligned}
$$

where $x=x_{1}-V t$.

The strains with the corresponding displacements are

$$
\begin{gathered}
\varepsilon_{y z}=\varepsilon_{z y}=\frac{1}{2}\left[\frac{b_{3}^{\|}}{2 \pi} \frac{\beta_{1} x}{x^{2}+\beta_{1}^{2} y^{2}}+\frac{b_{3}^{\perp}}{\pi} \frac{\beta_{2}^{2} R}{\rho V^{2}-\mu} \frac{3 x^{6}-12 x^{4} y^{2}+y^{6}+6\left(x^{7}-3 x^{5} y^{2}\right) \beta_{2}^{2}+2\left(x^{8}-3 x^{6} y^{2}\right) \beta_{2}^{4}}{\left(x^{2}+y^{2}\right)^{3}\left(1+\beta_{2}^{2} x\right)^{3}}\right] \\
\varepsilon_{z x}=\varepsilon_{x z}=\frac{1}{2}\left[-\frac{b_{3}^{\|}}{2 \pi} \frac{\beta_{1} y}{x^{2}+\beta_{1}^{2} y^{2}}+\right. \\
\frac{b_{3}^{\perp}}{\pi} \frac{\beta_{2}^{2} R}{\rho V^{2}-\mu} \frac{14 x^{3} y^{3}-2 x y^{5}+\left(-3 x^{6} y+38 x^{4} y^{3}+x^{2} y^{5}\right) \beta_{2}^{2}-8\left(x^{7} y-3 x^{5} y^{3}\right) \beta_{2}^{4}-2\left(x^{8} y-3 x^{6} y^{3}\right) \beta_{2}^{6}}{\left(x^{2}+y^{2}\right)^{3}\left(1+\beta_{2}^{2} x\right)^{4}} \\
w_{z y}=\frac{b_{3}^{\perp}}{2 \pi} \frac{x\left(x^{2}+y^{2}+2 \beta_{2}^{2} x y^{2}\right)}{\left(x^{2}+y^{2}\right)^{2}\left(1+\beta_{2}^{2} x\right)}-\frac{b_{3}^{\|}}{\pi} \frac{R}{K_{1}-K_{2}} \frac{x^{3}\left(x^{2}-3 \beta_{1}^{2} y^{2}\right)\left(\beta_{1}^{3}-\beta_{1}\right)}{\left(1+\beta_{2}^{2} x\right)\left(x^{2}+\beta_{1}^{2} y^{2}\right)^{3}} \\
w_{z x}=-\frac{b_{3}^{\perp}}{2 \pi} \frac{y\left(2 \beta_{2}^{2} x^{3}+y^{2}+4 \beta_{2}^{2} x y^{2}+x^{2}\left(1+2 \beta_{2}^{4} y^{2}\right)\right)}{\left(x^{2}+y^{2}\right)^{2}\left(1+\beta_{2}^{2} x\right)^{2}}+\frac{b_{3}^{\|}}{\pi} \frac{R}{K_{1}-K_{2}} \frac{x^{2} y\left(x^{2}+2 \beta_{2}^{2} x^{3}-2 \beta_{1}^{2} \beta_{2}^{2} x y^{2}-3 \beta_{1}^{2} y^{2}\right)\left(\beta_{1}^{3}-\beta_{1}\right)}{\left(1+\beta_{2}^{2} x\right)^{2}\left(x^{2}+\beta_{1}^{2} y^{2}\right)^{3}}
\end{gathered}
$$

The stresses are

$$
\begin{aligned}
& \sigma_{y z}=\sigma_{z y}=\frac{b \frac{1}{3}}{2 \pi}\left[\frac{2 \beta_{2}^{2} R \mu}{\rho V^{2}-\mu} \frac{3 x^{6}-12 x^{4} y^{2}+y^{6}+6\left(x^{7}-3 x^{5} y^{2}\right) \beta_{2}^{2}+2\left(x^{8}-3 x^{6} y^{2}\right) \beta_{2}^{4}}{\left(x^{2}+y^{2}\right)^{3}\left(1+\beta_{2} 2 x\right)^{3}}+\frac{R x\left(x^{2}+y^{2}+2 \beta_{2}^{2} x y^{2}\right)}{\left(x^{2}+y^{2}\right)^{2}\left(1+\beta_{2}{ }^{2} x\right)}\right] \\
& +\frac{b_{3}^{\|}}{2 \pi}\left[\frac{\mu \beta_{1} x}{x^{2}+\beta_{1}^{2} y^{2}}-\frac{2 R^{2}}{K_{1}-K_{2}} \frac{x^{3}\left(x^{2}-3 \beta_{1}^{2} y^{2}\right)\left(\beta_{1}^{3}-\beta_{1}\right)}{\left(1+\beta_{2}^{2} x\right)\left(x^{2}+\beta_{1}^{2} y^{2}\right)^{3}}\right] \\
& \sigma_{z x}=\sigma_{x z}=\frac{b_{3}^{\frac{1}{2}} R}{2 \pi}\left[\frac{2 \beta_{2}^{2} \mu}{\rho V^{2}-\mu} \frac{\left.14 x^{3} y^{3}-2 x y^{5}+\left(-3 x^{6} y+38 x^{4} y^{3}+x^{2} y^{5}\right) \beta_{2}^{2}-8\left(x^{7} y-3 x^{5} y^{3}\right) \beta_{2}^{4}-2\left(x^{8} y-3 x^{6} y^{3}\right) \beta_{2}^{6}\right)}{\left(x^{2}+y^{2}\right)^{3}\left(1+\beta_{2}^{2} x\right)^{4}}\right. \\
& \left.-\frac{y\left(2 \beta_{2}^{2} x^{3}+y^{2}+4 \beta_{2}^{2} x y^{2}+x^{2}\left(1+2 \beta_{2}^{4} y^{2}\right)\right)}{\left(x^{2}+y^{2}\right)^{2}\left(1+\beta_{2}^{2} x\right)^{2}}\right]+\frac{b_{3}^{\|}}{2 \pi}\left[\frac{2 R^{2}}{K_{1}-K_{2}} \frac{x^{2} y\left(x^{2}+2 \beta_{2}^{2} x^{3}-2 \beta_{1}^{2} \beta_{2}^{2} x y^{2}-3 \beta_{1}^{2} y^{2}\right)\left(\beta_{1}^{3}-\beta_{1}\right)}{\left(1+\beta_{2}^{2} x\right)^{2}\left(x^{2}+\beta_{1}^{2} y^{2}\right)^{3}}\right. \\
& \left.-\frac{\mu \beta_{1} y}{x^{2}+\beta_{1}^{2} y^{2}}\right] \\
& H_{z y}=\frac{b_{3}^{\perp}}{2 \pi}\left[\frac{2 \beta_{2}^{2} R^{2}}{\rho V^{2}-\mu} \frac{3 x^{6}-12 x^{4} y^{2}+y^{6}+6\left(x^{7}-3 x^{5} y^{2}\right) \beta_{2}^{2}+2\left(x^{8}-3 x^{6} y^{2}\right) \beta_{2}^{4}}{\left(x^{2}+y^{2}\right)^{3}\left(1+\beta_{2}^{2} x\right)^{3}}+\left(K_{1}-K_{2}\right) \frac{x\left(x^{2}+y^{2}+2 \beta_{2}^{2} x y^{2}\right)}{\left(x^{2}+y^{2}\right)^{2}\left(1+\beta_{2}^{2} x\right)}\right] \\
& +\frac{b_{3}^{\|} R}{2 \pi}\left[\frac{\beta_{1} x}{x^{2}+\beta_{1}^{2} y^{2}}-\frac{2 x^{3}\left(x^{2}-3 \beta_{1}^{2} y^{2}\right)\left(\beta_{1}^{3}-\beta_{1}\right)}{\left(1+\beta_{2}^{2} x\right)\left(x^{2}+\beta_{1}^{2} y^{2}\right)^{3}}\right] \\
& H_{z x}=\frac{b \frac{\perp}{2 \pi}}{2 \pi}\left[\frac{2 \beta_{2}^{2} R}{\rho V^{2}-\mu} \frac{14 x^{3} y^{3}-2 x y^{5}+\left(-3 x^{6} y+38 x^{4} y^{3}+x^{2} y^{5}\right) \beta_{2}{ }^{2}-8\left(x^{7} y-3 x^{5} y^{3}\right) \beta_{2}^{4}-2\left(x^{8} y-3 x^{6} y^{3}\right) \beta_{2}^{6}}{\left(x^{2}+y^{2}\right)^{3}\left(1+\beta_{2}^{2} x\right)^{4}}\right. \\
& \left.-\left(K_{1}-K_{2}\right) \frac{y\left(2 \beta_{2}^{2} x^{3}+y^{2}+4 \beta_{2}^{2} x y^{2}+x^{2}\left(1+2 \beta_{2}^{4} y^{2}\right)\right)}{\left(x^{2}+y^{2}\right)^{2}\left(1+\beta_{2}^{2} x\right)^{2}}\right]+\frac{b_{3}^{\|} R}{2 \pi}\left[\frac{2 x^{2} y\left(x^{2}+2 \beta_{2}^{2} x^{3}-2 \beta_{1}^{2} \beta_{2}^{2} x y^{2}-3 \beta_{1}^{2} y^{2}\right)\left(\beta_{1}^{3}-\beta_{1}\right)}{\left(1+\beta_{2}^{2} x\right)^{2}\left(x^{2}+\beta_{1}^{2} y^{2}\right)^{3}}\right. \\
& \left.-\frac{\beta_{1} y}{x^{2}+\beta_{1}^{2} y^{2}}\right]
\end{aligned}
$$

For cubic or hexagonal quasicrystals, the solutions are obtained by replacing $\mu,\left(K_{1}-K_{2}\right)$ and $R$ by $C_{44}, K_{44}$ and $R_{44}$ or by $C_{44}, K_{2}$ and $R_{3}$ respectively in the above equations. It is pointed out that the solution is composed of three parts, namely, wave propagation, diffusion motion and their interaction. The part of the phonon field is governed by wave propagation, and the part of the phason field is governed by diffusion motion. Consequently, the difference between the present solution and the 
dynamic solution of the based on Bak's argument lies in the diffuse effect. Let $V=0$, the present solution can be reduced to the static dislocation solution. The solution reveals that the phason field impedes dislocation.

For illustrations, the case of $V=0.1 c, c=\sqrt{\mu / \rho}$ is depicted in Figures 3-6. In the numerical computation we take $b_{3}^{\perp}=0.8 b_{3}^{\|}, \rho=4.1 \mathrm{~kg} / \mathrm{cm}^{3}, \mu=70 \mathrm{GPa}, K_{1}=72 \mathrm{MPa}, K_{2}=-37 \mathrm{MPa}$, $R / \mu=0.004, \Gamma_{w}=1 / \kappa=4.8 \times 10^{-19} \mathrm{~m}^{3} \mathrm{gs} / \mathrm{kg}[69]$.

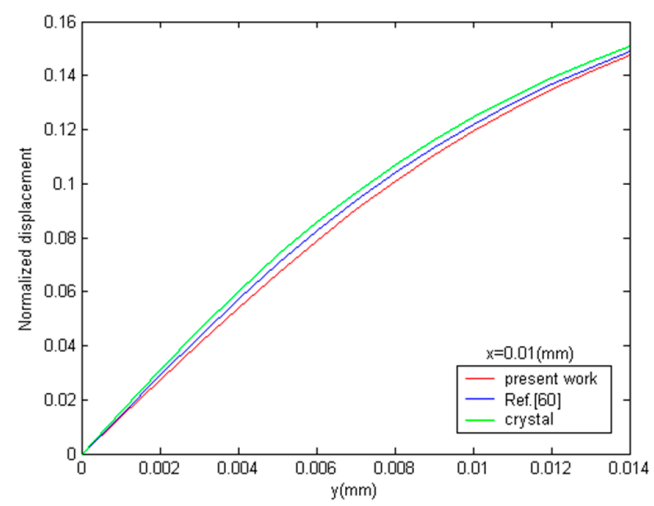

Figure 3. Variations of $u_{z} / b_{3}^{\|}$with $y(x=0.01 \mathrm{~mm})$.

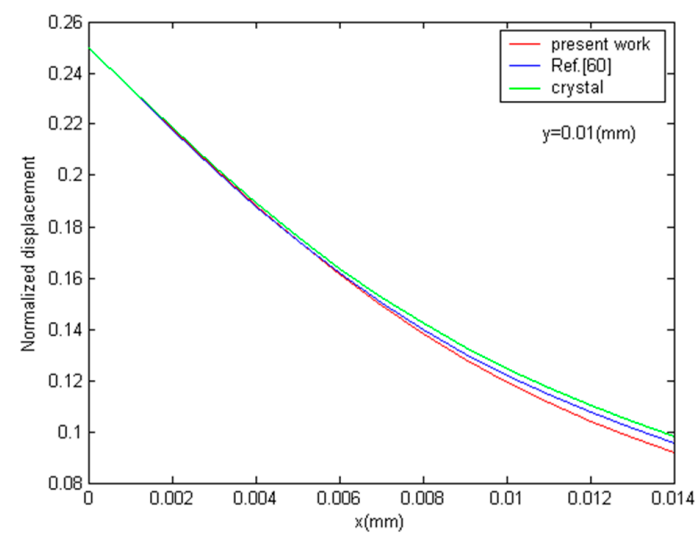

Figure 4. Variations of $u_{z} / b_{3}^{\|}$with $x(y=0.01 \mathrm{~mm})$.

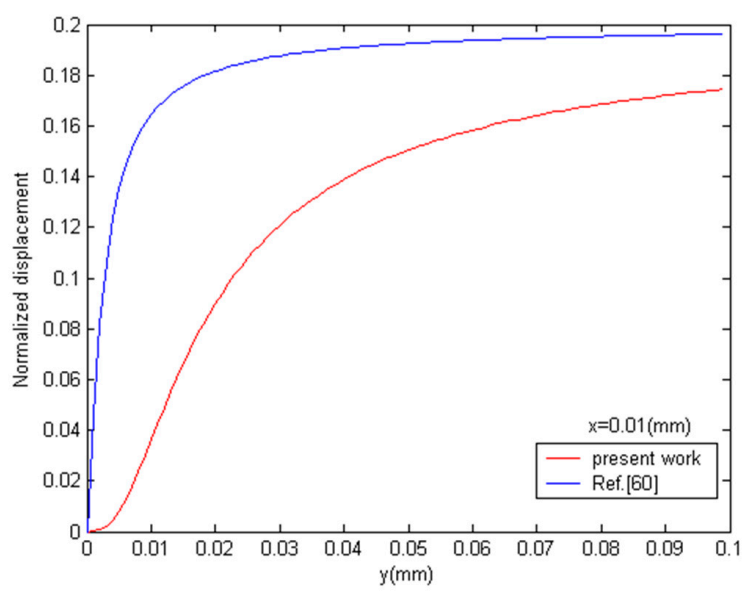

Figure 5. Variations of $w_{z} / b_{3}^{\|}$with $y(x=0.01 \mathrm{~mm})$. 


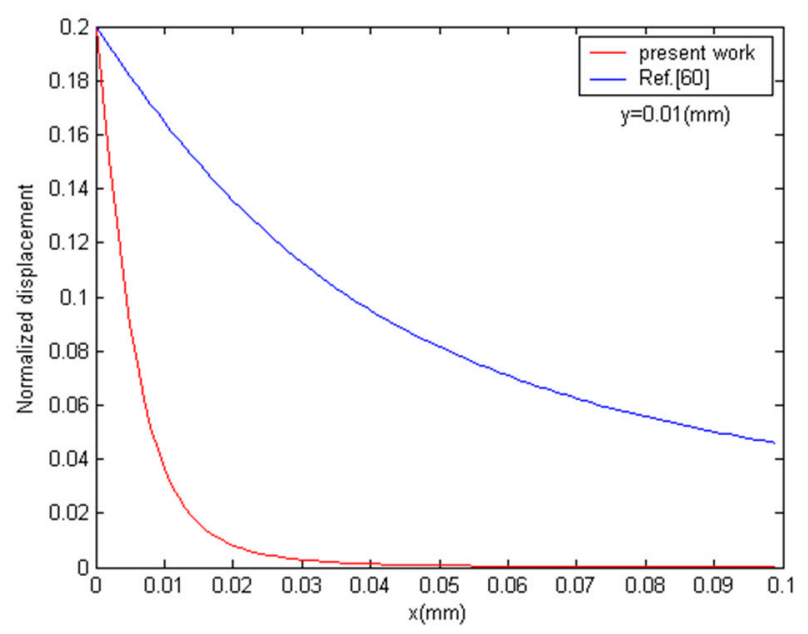

Figure 6. Variations of $w_{z} / b_{3}^{\|}$with $x(y=0.01 \mathrm{~mm})$.

\subsection{Application 2 of Elasto-/Hydro-Dynamics—Dynamic Propagating Crack and Solutions of} Two-Dimensional Decagonal Quasicrystals

On the basis of the preceding elasto-/hydro-dynamics formulation we consider two-dimensional quasicrystals in this section. By using the dynamic Equation (57), we are able to formulate two-dimensional elasto-/hydro-dynamics of quasicrystals. For illustration of its applications, we only present some solutions in this section.

Because two-dimensional decagonal quasicrystals occupy an important position in all quasicrystals observed to date, herein we consider only the two-dimensional decagonal quasicrystals. Referring to Figure 7, suppose the periodic direction is the $z$ axis and a Griffith crack penetrates along the $z$ axis in the solids. If the elastic field is induced by the uniform tensile stress at the upper and lower surfaces of the specimen, it is obvious that all variables of the elastic field are independent of $z$, i.e., $\partial / \partial z=0$. In this case the stress-strain relations can be written in

$$
\begin{aligned}
& \sigma_{x x}=L\left(\varepsilon_{x x}+\varepsilon_{y y}\right)+2 M \varepsilon_{x x}+R\left(w_{x x}+w_{y y}\right) \\
& \sigma_{y y}=L\left(\varepsilon_{x x}+\varepsilon_{y y}\right)+2 M \varepsilon_{y y}-R\left(w_{x x}+w_{y y}\right) \\
& \sigma_{x y}=\sigma_{y x}=2 M \varepsilon_{x y}+R\left(w_{y x}-w_{x y}\right) \\
& H_{x x}=K_{1} w_{x x}+K_{2} w_{y y}+R\left(\varepsilon_{x x}-\varepsilon_{y y}\right) \\
& H_{y y}=K_{1} w_{y y}+K_{2} w_{x x}+R\left(\varepsilon_{x x}-\varepsilon_{y y}\right) \\
& H_{x y}=K_{1} w_{x y}-K_{2} w_{y x}-2 R \varepsilon_{x y} \\
& H_{y x}=K_{1} w_{y x}-K_{2} w_{x y}+2 R \varepsilon_{x y}
\end{aligned}
$$

where $L=C_{12}, M=\left(C_{11}-C_{12}\right) / 2$ denote the elastic constants of the phonon field, $K_{1}$ and $K_{2}$ are the elastic constants of the phason field, $R$ is the coupling elastic constant of the phonon-phason.

To substitute Equations (98) into (57), one obtains the motion equations of decagonal quasicrystals as follows

$$
\begin{gathered}
\frac{\partial^{2} u_{x}}{\partial t^{2}}=c_{1}^{2} \frac{\partial^{2} u_{x}}{\partial x^{2}}+\left(c_{1}^{2}-c_{2}^{2}\right) \frac{\partial^{2} u_{y}}{\partial x \partial y}+c_{2}^{2} \frac{\partial^{2} u_{x}}{\partial y^{2}}+c_{3}^{2}\left(\frac{\partial^{2} w_{x}}{\partial x^{2}}+2 \frac{\partial^{2} w_{y}}{\partial x \partial y}-\frac{\partial^{2} w_{x}}{\partial y^{2}}\right) \\
\frac{\partial^{2} u_{y}}{\partial t^{2}}=c_{2}^{2} \frac{\partial^{2} u_{y}}{\partial x^{2}}+\left(c_{1}^{2}-c_{2}^{2}\right) \frac{\partial^{2} u_{x}}{\partial x \partial y}+c_{1}^{2} \frac{\partial^{2} u_{y}}{\partial y^{2}}+c_{3}^{2}\left(\frac{\partial^{2} w_{y}}{\partial x^{2}}-2 \frac{\partial^{2} w_{x}}{\partial x \partial y}-\frac{\partial^{2} w_{y}}{\partial y^{2}}\right) \\
\frac{\partial w_{x}}{\partial t}=d_{1}^{2}\left(\frac{\partial^{2} w_{x}}{\partial x^{2}}+\frac{\partial^{2} w_{x}}{\partial y^{2}}\right)+d_{2}^{2}\left(\frac{\partial^{2} u_{x}}{\partial x^{2}}-2 \frac{\partial^{2} u_{y}}{\partial x \partial y}-\frac{\partial^{2} u_{x}}{\partial y^{2}}\right)
\end{gathered}
$$




$$
\frac{\partial w_{y}}{\partial t}=d_{1}^{2}\left(\frac{\partial^{2} w_{y}}{\partial x^{2}}+\frac{\partial^{2} w_{y}}{\partial y^{2}}\right)+d_{2}^{2}\left(\frac{\partial^{2} u_{y}}{\partial x^{2}}+2 \frac{\partial^{2} u_{x}}{\partial x \partial y}-\frac{\partial^{2} u_{y}}{\partial y^{2}}\right)
$$

where

$$
c_{1}=\sqrt{\frac{L+2 M}{\rho}}, c_{2}=\sqrt{\frac{M}{\rho}}, c_{3}=\sqrt{\frac{R}{\rho}}, d_{1}=\sqrt{\frac{K_{1}}{\kappa}} \text { and } d_{2}=\sqrt{\frac{R}{\kappa}}, d_{3}=\sqrt{\frac{K_{2}}{\kappa}}
$$

The constants $c_{1}, c_{2}$ and $c_{3}$ are the elastic wave speeds; $d_{1}^{2}, d_{2}^{2}$ and $d_{3}^{2}$ are the diffusive coefficients.

As shown in Figure 7, the decagonal quasicrystal with a Griffith crack is a rectangular specimen with a central crack of length $2 a(t)$ subjected to a dynamic or static tensile stress at its ends; $a(t)$ is the crack length which is a function of time. Due to the symmetry of the specimen only the upper right quarter is considered. For dynamic initial growth of the crack, suppose that the crack is stable, i.e., $a(t)=a_{0}$ is a constant; but for fast propagation of the crack, $a(t)$ is not a constant, instead it is unstable. Here we only consider dynamic initiation of crack growth, then fast propagation of the crack is omitted. The content can be seen in $[23,62,63]$.

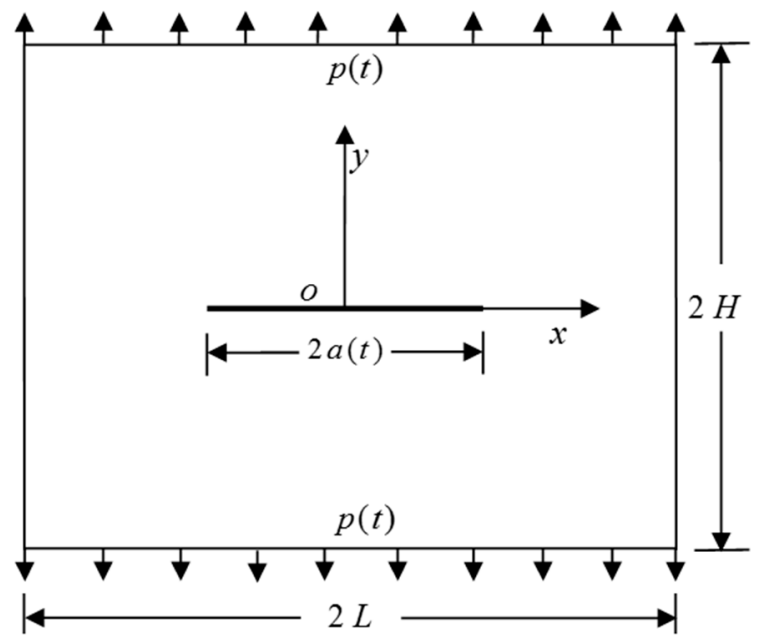

Figure 7. The Griffith specimen.

Due to symmetry of the specimen, it is sufficient to analyze the elastic field in the upper right quarter. Under such circumstances, if the upper right quarter is considered then the boundary conditions can be stated as follows

$$
\begin{gathered}
u_{x}=0, \sigma_{y x}=0, w_{x}=0, H_{y x}=0 \text { on } x=0 \text { for } 0 \leq y \leq H \\
\sigma_{x x}=0, \sigma_{y x}=0, H_{x x}=0, H_{y x}=0 \text { on } x=L \text { for } 0 \leq y \leq H \\
\sigma_{y y}=p(t), \sigma_{x y}=0, H_{y y}=0, H_{x y}=0 \text { on } y=H \text { for } 0 \leq x \leq H \\
\sigma_{y y}=0, \sigma_{x y}=0, H_{y y}=0, H_{x y}=0 \text { on } y=0 \text { for } 0 \leq x \leq a(t) \\
u_{y}=0, \sigma_{x y}=0, w_{y}=0, H_{x y}=0 \text { on } y=0 \text { for } a(t) \leq x \leq L
\end{gathered}
$$

in which $p(t)=p_{0} f(t)$ is a dynamic load, $p_{0}=$ const is use to denote the stress.

The initial conditions are that the initial displacements and velocities of the phonon field and phason field are equal to zero, i.e.,

$$
\begin{array}{ll}
\left.u_{x}(x, y, t)\right|_{t=0}=0 & \left.u_{y}(x, y, t)\right|_{t=0}=0 \\
\left.w_{x}(x, y, t)\right|_{t=0}=0 & \left.w_{y}(x, y, t)\right|_{t=0}=0 \\
\left.\frac{\partial u_{x}(x, y, t)}{\partial t}\right|_{t=0}=0 & \left.\frac{\partial u_{y}(x, y, t)}{\partial t}\right|_{t=0}=0
\end{array}
$$


We shall solve the problem by using the finite difference method. To this end, the field variables and boundary-initial conditions will be expressed in terms of the displacements. This can be done through the constitutive Equation (98). For computation, the mass density of two-dimensional decagonal Al-Ni-Co quasicrystals $\rho=4.186 \times 10^{-3} \mathrm{~g} \cdot \mathrm{mm}^{-3}$ is used. The phonon elastic moduli are $C_{11}=2.3433, C_{12}=0.5741\left(10^{12} \mathrm{dyn} / \mathrm{cm}^{2}=10^{2} \mathrm{GPa}\right)$ obtained by resonant ultrasound spectroscopy [17]. The elastic constants of the phason field are $K_{1}=1.22 \mathrm{Gpa}$ and $K_{2}=0.24 \mathrm{Gpa}$ estimated by Monto-Carlo simulation [21], and $\Gamma_{w}=1 / \kappa=4.8 \times 10^{-19} \mathrm{~m}^{3} \mathrm{gs} / \mathrm{kg}$. The coupling constant $R$ of the quasicrystal is taken to be $R / M=0.01$, and $R / M=0$ for the uncoupled case in crystals.

When the specimen with a invariable crack is loaded by a varying applied load $p(t)=p_{0} f(t)$, in which $f(t)$ is the Heaviside function, the coupled phonon and phason reveals the distinctive physical properties of the quasicrystals in contrast with the periodic crystals. The normalized dynamics stress intensity factor $K_{\mathrm{I}}(t) / \sqrt{\pi a_{0}} p_{0}$ is used, and the numerical results are plotted in Figure 8 . It is worth noting that one curve indicates the quasicrystal with $R / M=0.01$ and the other indicates the periodic crystals with $R / M=0$. Due to the coupling effect of the phonon and phason, the normalized dynamics stress intensity factor between quasicrystals and classical crystals is quite different. In the figure, $t_{0}=2.6735 \mu \mathrm{s}$ is the time that the wave travels from the external boundary to the crack surface. So the velocity of the wave propagation can be computed as $v_{0}=H / t_{0}=7.4807 \mathrm{~km} / \mathrm{s}$, which is completely in conformity with the longitudinal wave speed $c_{1}=\sqrt{(L+2 M) / \rho}$ of the phonon field. This fact declares that the phonon wave propagation is dominant in coupling of the wave propagation-motion of diffusion, and there are oscillations in the figure of the stress intensity factor that are especially clear. The oscillations declare that the reflection and diffraction between waves come from the crack surface and the specimen boundary surfaces. The oscillations may be greatly influenced by the specimen geometry and the material constants.

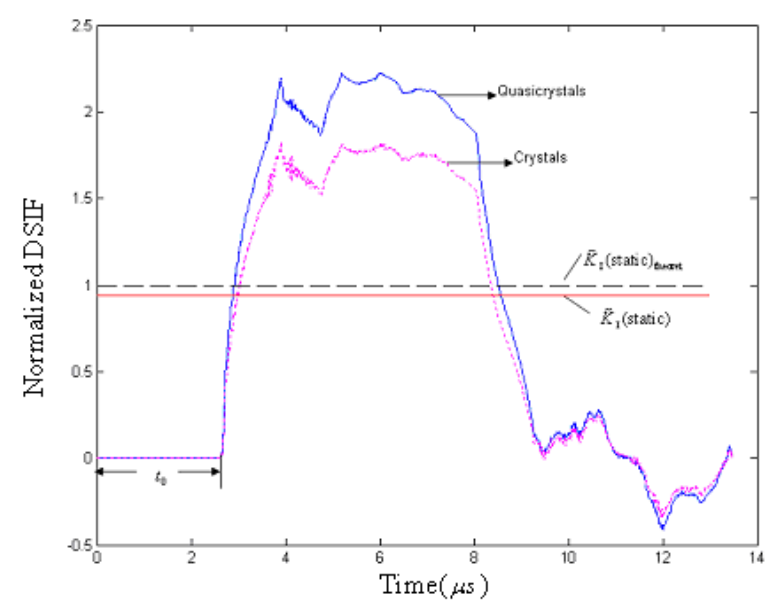

Figure 8. Variations of $K_{\mathrm{I}}(t) / \sqrt{\pi a_{0}} p_{0}$ with time.

\subsection{Application 3 of Elasto-/Hydro-Dynamics-Dynamic Crack Propagation of Icosahedral Quasicrystals}

\subsubsection{A Cracked Specimen of Icosahedral Quasicrystals of Plane Problem Based on FDM}

Referring to Figure 9, consider a centered crack specimen of icosahedral QCs of plane problem. Suppose a Griffith crack penetrates through the specimen of icosahedral QCs along the quasi-periodic direction, i.e., the $z$ axis direction. Consider a deformation of the specimen induced by a uniform tensile stress at upper and lower surfaces of icosahedral QCs such that it is obvious that all variables of elastic field are independent of $z$, i.e., $\frac{\partial}{\partial z}=0$. Then all the equations will be simple. 


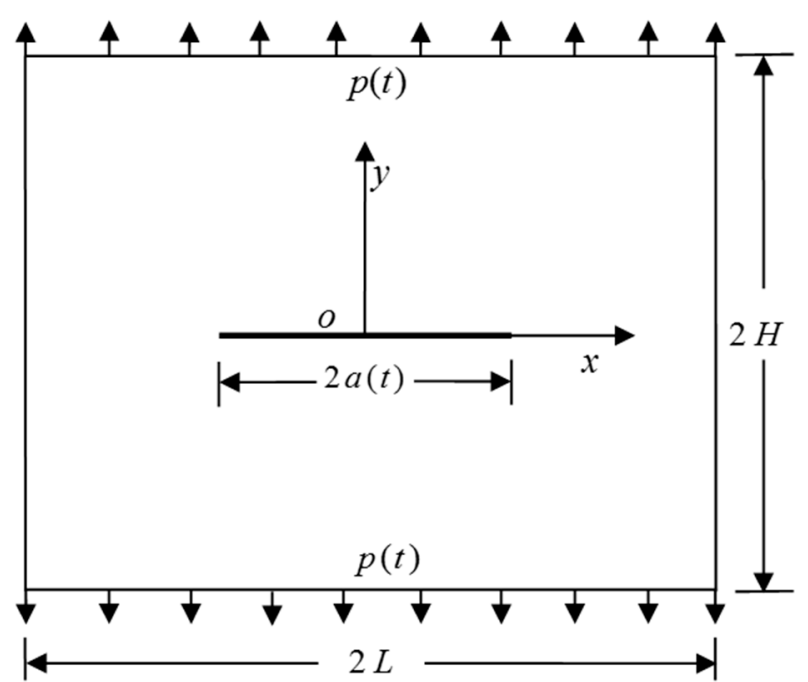

Figure 9. The specimen for icosahedral quasicrystals (QCs) with a Griffith crack.

Let us regard displacements of equilibrium as the unknown variables of the governing differential equations for a two-dimensional problem, then the equation can be written easily such that

$$
\begin{gathered}
\frac{\partial^{2} u_{x}}{\partial t^{2}}=c_{1}^{2} \frac{\partial^{2} u_{x}}{\partial x^{2}}+\left(c_{1}^{2}-c_{2}^{2}\right) \frac{\partial^{2} u_{y}}{\partial x \partial y}+c_{2}^{2} \frac{\partial^{2} u_{x}}{\partial y^{2}}+c_{3}^{2}\left(\frac{\partial^{2} w_{x}}{\partial x^{2}}+2 \frac{\partial^{2} w_{y}}{\partial x \partial y}-\frac{\partial^{2} w_{x}}{\partial y^{2}}\right) \\
\frac{\partial^{2} u_{y}}{\partial t^{2}}=c_{2}^{2} \frac{\partial^{2} u_{y}}{\partial x^{2}}+\left(c_{1}^{2}-c_{2}^{2}\right) \frac{\partial^{2} u_{x}}{\partial x \partial y}+c_{1}^{2} \frac{\partial^{2} u_{y}}{\partial y^{2}}+c_{3}^{2}\left(\frac{\partial^{2} w_{y}}{\partial x^{2}}-2 \frac{\partial^{2} w_{x}}{\partial x \partial y}-\frac{\partial^{2} w_{y}}{\partial y^{2}}\right) \\
\frac{\partial^{2} u_{z}}{\partial t^{2}}=c_{2}^{2} \nabla^{2} u_{z}+c_{3}^{2}\left(\frac{\partial^{2} w_{x}}{\partial x^{2}}-\frac{\partial^{2} w_{x}}{\partial y^{2}}-2 \frac{\partial^{2} w_{y}}{\partial x \partial y}+\nabla^{2} w_{z}\right) \\
\frac{\partial w_{x}}{\partial t}=d_{1} \nabla^{2} w_{x}+d_{2}\left(\frac{\partial^{2}}{\partial x^{2}}-\frac{\partial^{2}}{\partial y^{2}}\right) w_{z}+d_{3}\left(\frac{\partial^{2} u_{x}}{\partial x^{2}}-2 \frac{\partial^{2} u_{y}}{\partial x \partial y}-\frac{\partial^{2} u_{x}}{\partial y^{2}}+\frac{\partial^{2} u_{z}}{\partial x^{2}}-\frac{\partial^{2} u_{z}}{\partial y^{2}}\right) \\
\frac{\partial w_{y}}{\partial t}=d_{1} \nabla^{2} w_{y}-d_{2} \frac{\partial^{2} w_{z}}{\partial x \partial y}+d_{3}\left(\frac{\partial^{2} u_{y}}{\partial x^{2}}+2 \frac{\partial^{2} u_{x}}{\partial x \partial y}-\frac{\partial^{2} u_{y}}{\partial y^{2}}-2 \frac{\partial^{2} u_{z}}{\partial x \partial y}\right) \\
\frac{\partial^{2} w_{z}}{\partial t^{2}}=\left(d_{1}-d_{2}\right) \nabla^{2} w_{z}+d_{2}\left(\frac{\partial^{2} w_{x}}{\partial x^{2}}-\frac{\partial^{2} w_{x}}{\partial y^{2}}-2 \frac{\partial^{2} w_{y}}{\partial x \partial y}\right)+d_{3} \nabla^{2} u_{z}
\end{gathered}
$$

where $\nabla^{2}$ is two-dimensional Laplacian operator, and $c_{1}=\sqrt{\frac{\lambda+2 \mu}{\rho}}, c_{2}=\sqrt{\frac{\mu}{\rho}}, c_{3}=\sqrt{\frac{R}{\rho}}, d_{1}=\frac{K_{1}}{\kappa}$, $d_{2}=\frac{K_{2}}{\kappa}$ and $d_{3}=\frac{R}{\kappa}$. It needs to be noticed that the known constants $c_{1}, c_{2}$ and $c_{3}$ represent elastic wave speeds of the phonon fields, while $d_{1}, d_{2}$ and $d_{3}$ are diffusive coefficients, and do not represent wave speeds. The length of the specimen is $2 L$ and the height is $2 H$, and $2 a(t)$ is the length of the crack. Due to symmetry of the specimen, it is sufficient to analyze the elastic field in the upper right quarter. Under such circumstances, if the upper right quarter is considered then the boundary conditions can be stated as follows

$$
\begin{gathered}
u_{x}=0, \sigma_{y x}=0, \sigma_{z x}=0, w_{x}=0, H_{y x}=0, H_{z x}=0 x=0,0 \leq y \leq H \\
\sigma_{x x}=0, \sigma_{y x}=0, \sigma_{z x}=0, H_{x x}=0, H_{y x}=0, H_{z x}=0 x=L, 0 \leq y \leq H \\
\sigma_{y y}=p(t), \sigma_{x y}=0, \sigma_{z y}=0, H_{y y}=0, H_{x y}=0, H_{z y}=0 y=H, 0 \leq x \leq H \\
\sigma_{y y}=0, \sigma_{x y}=0, \sigma_{z y}=0, H_{y y}=0, H_{x y}=0, H_{z y}=0 y=0,0 \leq x \leq a(t)
\end{gathered}
$$




$$
u_{y}=0, \sigma_{x y}=0, \sigma_{z y}=0, w_{y}=0, H_{x y}=0, H_{z y}=0 y=0, a(t)<y \leq L
$$

where $p(t)$ is used for simulating a dynamic loading.

Suppose the initial displacements and velocities of the phonon field and phason field are equal to zero, i.e.,

$$
\left.u_{i}(x, y, t)\right|_{t=0}=0,\left.w_{i}(x, y, t)\right|_{t=0}=0,\left.\frac{\partial u_{i}}{\partial t}(x, y, t)\right|_{t=0}=0,(i=x, y, z)
$$

In order to obtain the numerical results, we now pay attention to investigating the effect of the phonon and phason fields in the icosahedral Al-Pd-Mn QCs. The related parameters are used, in which the density $\rho=5.1 \mathrm{~g} / \mathrm{cm}^{3}$ and the elastic constants $\lambda=75(\mathrm{GPa}), \mu=65(\mathrm{GPa})$ for the phonon fields [69], meanwhile the elastic constants $K_{1}=300 \mathrm{MPa}, K_{2}=-45 \mathrm{MPa}$ for phason fields [70] and the relevant constant of diffusion coefficient $\Gamma_{w}=1 / \kappa=4.8 \times 10^{-10} \mathrm{~cm}^{3} \cdot \mu \mathrm{s} / \mathrm{g}$ for phason fields. The phonon-phason coupling constant has not been measured for icosahedral QCs so far. In view of contrasting with the measured parameters of the decagonal quasicrystals, we take $R / \mu=0.01$ for icosahedral QCs too. Below we graphically display the results.

In the following section we take the related parameters $H=20 \mathrm{~mm}, L=52 \mathrm{~mm}$, the initial length $a_{0}=12 \mathrm{~mm}$ of the crack. Firstly, we will discuss the dynamic stress intensity factor in the dynamic loadings for: (a) different Poisson coefficients while the density of material and the elastic constants of phason field remain unchanged; (b) different the elastic constants of the phason field as a fixed value $K_{2}=-45 \mathrm{MPa}$, and $K_{1}$ takes different values while Poisson coefficients remain unchanged. Please see Table 1:

Table 1. Mechanical properties of icosahedral quasicrystals $[16,17,21,69,70]$.

\begin{tabular}{cccc}
\hline & $\lambda$ (GPa) & $\mu$ (GPa) & $\nu$ \\
\hline Al-Li-Cu & 30.4 & 40.9 & 0.213 \\
Al-Cu-Fe-Ru & 48.4 & 57.9 & 0.228 \\
Al-Pa-Mn & 74.9 & 72.4 & 0.254 \\
\hline
\end{tabular}

This difference in Figure 10a can be summarized as: the change of wave is caused by the change of $v$ value, which can lead to the refraction and reflection of stress wave with different velocity in the solid. So it is predictable that the difference will become very remarkable when the loading sustains. Assuming the phonon field of material constants $\lambda=75, \mu=65$ (GPa), the dynamic stress intensity factors are depicted in the Figure 10b. It can be seen that when the elastic constant of the phason field as a fixed value $K_{2}=-45 \mathrm{MPa}$, and $K_{1}$ takes different values, the dynamic stress intensity factors fluctuate at the start of a period of time, the dynamic stress intensity factor decreases with the increase of $K_{1}$, and after a period of time, the dynamic stress intensity factor increases with the increase of $K_{1}$. Also if $K_{1}$ is taken as a fixed value, and $K_{2}$ takes different values, the dynamic stress intensity factors are hardly altered. Thus it can be declared that $K_{1}$ occupies a central role in the deformation of QCs, and this effect cannot be ignored. In addition, we can infer that in three-dimensional icosahedral QCs, the two elastic constants of phason field, $K_{1}$ plays a leading role. 


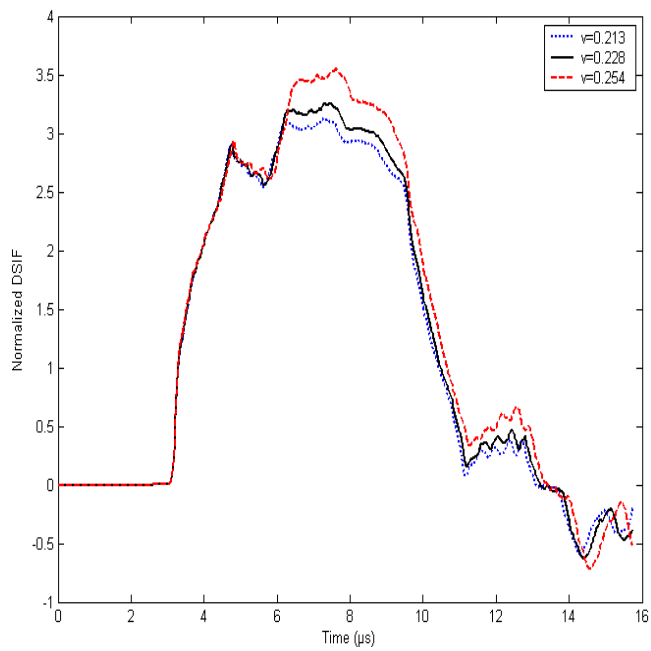

(a)

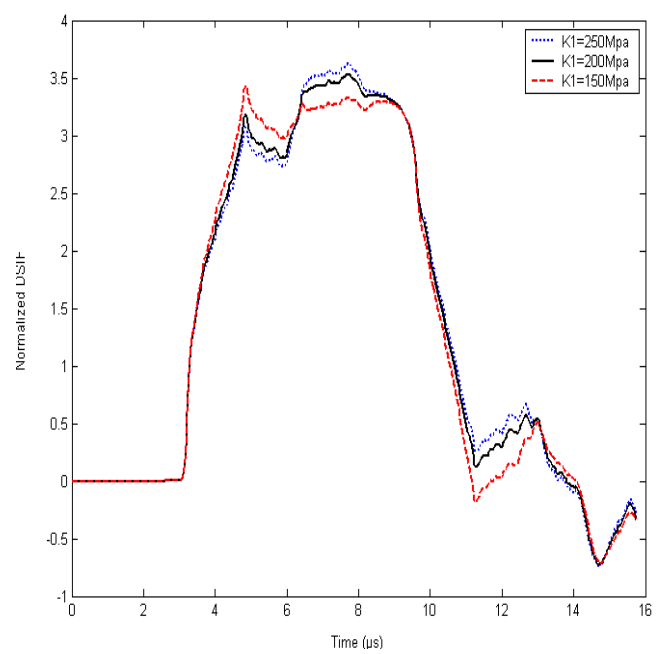

(b)

Figure 10. Normalized dynamic stress intensity factors for different Poisson coefficients (a); and for different $K_{1}(\mathbf{b})$.

The fast crack propagation also belongs to nonlinear problem in the deformation of QCs, in which the movement rule of crack is unknown in advance. In the field of fracture mechanics research, the researchers always make some simplifying assumptions to the movement rule of crack when they give the analysis for the propagation of the crack. We suppose that the crack extends along the $x$-axial direction and is related to $y$-axial with symmetry. The parameters of the specimen are given by $L=50 \mathrm{~mm}, H=20 \mathrm{~mm}$, and the initial length of the crack is $a_{0}=12 \mathrm{~mm}$ and the parameters of material can be seen in the foregoing section, and $p_{0}=80 \mathrm{Mpa}$. Suppose the pull stress in the vicinity of crack tip is the parameter to control the fracture of QCs. By applying stress criterion, the fast crack propagation of icosahedral QCs specimen with a center crack is discussed in this section. In the mathematical iteration, the pull stress perpendicular to the crack surface of a point in the vicinity of the crack tip is calculated at any time. When its value is beyond stress threshold $\sigma_{c}$ of the fracture, the crack expands forward a grid. Here the calculation is based on $\sigma_{c}=450,550,650(\mathrm{MPa})$. The results can be reflected by Figures 11-13.

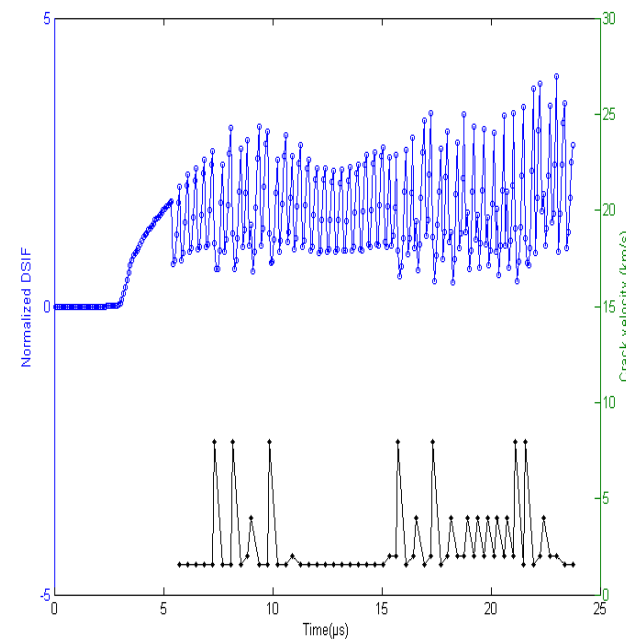

(a)
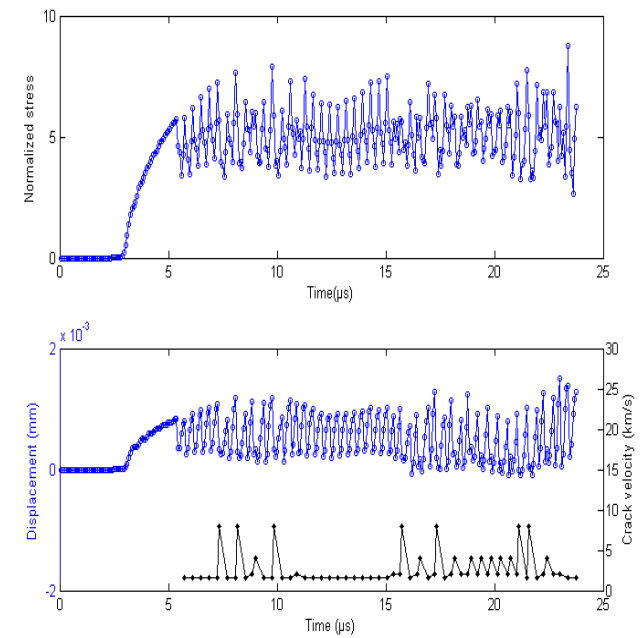

(b)

Figure 11. Dynamic stress intensity factor ( $\overrightarrow{0}$ ) and crack propagation speed ( $\rightarrow$ ) for $\sigma_{c}=450 \mathrm{MPa}(\mathbf{a})$; and the stress and displacement near the crack tip for $\sigma_{\mathcal{c}}=450 \mathrm{MPa}(\mathbf{b})$. 


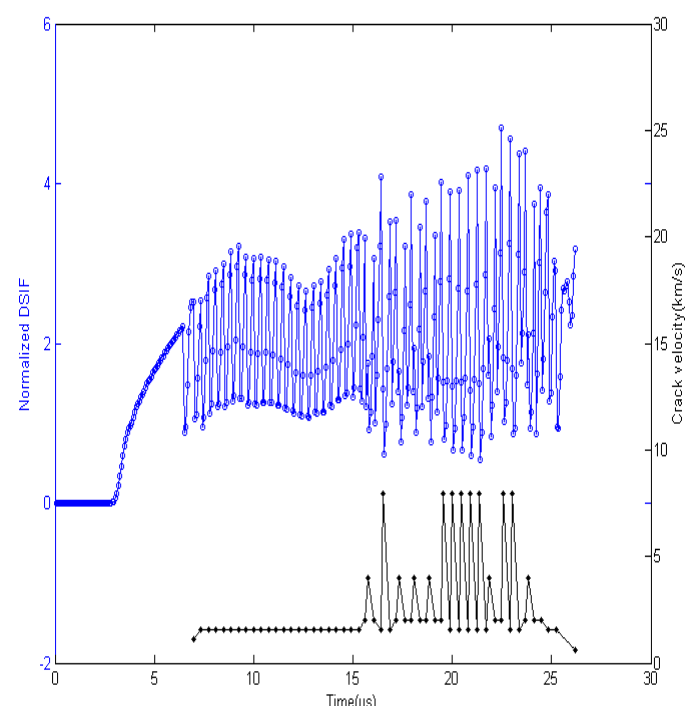

(a)
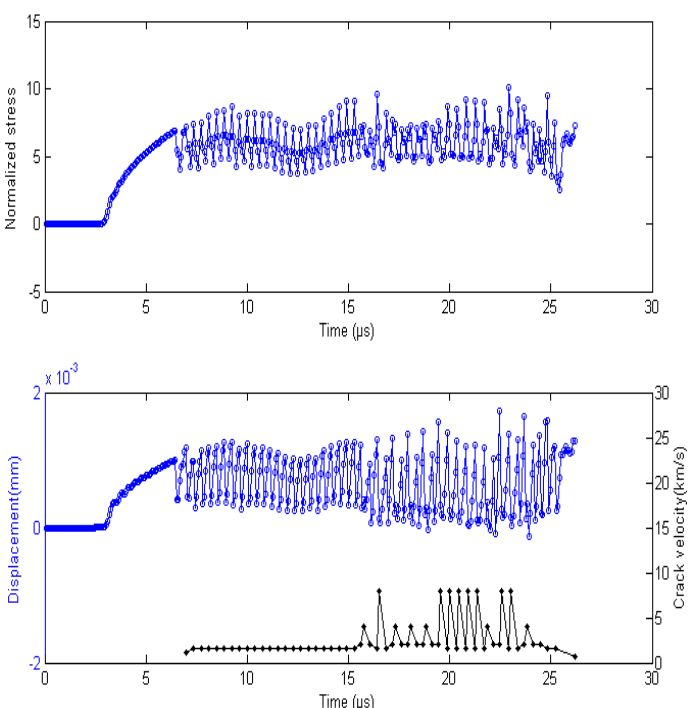

(b)

Figure 12. Dynamic stress intensity factor ( $\vec{\sigma}$ ) and crack propagation speed ( $*$ ) for $\sigma_{c}=550 \mathrm{MPa}(\mathbf{a})$; and the stress and displacement near the crack tip for $\sigma_{\mathcal{c}}=550 \mathrm{MPa}(\mathbf{b})$.

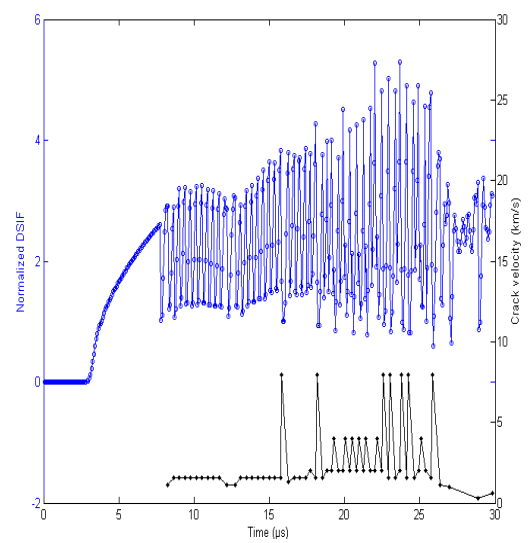

(a)
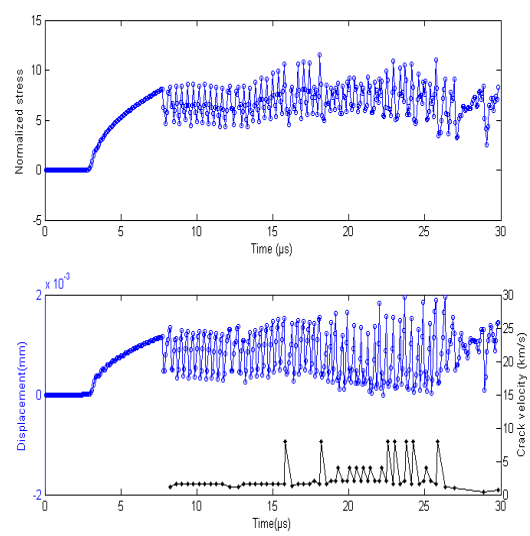

(b)

Figure 13. Dynamic stress intensity factor $(-)$ ) and crack propagation speed ( $\rightarrow$ ) for $\sigma_{c}=650 \mathrm{MPa}(\mathbf{a})$; and the stress and displacement near the crack tip for $\sigma_{\mathcal{c}}=650 \mathrm{MPa}(\mathbf{b})$.

The results can be seen in these figures, which reveal: which reveal: (1) The speed rate of crack propagation along with the increase of $\sigma_{c}$ becomes smooth, and has a tendency to decrease; (2) In the crack propagation, the crack opening displacement near the crack tip has the same tendency with the stress; (3) In the process of simulation, for cracks found near the plate location, the stress intensity factor and stress suddenly become large (we only describe the crack extension to have a short distance from the plate end location), the extension rate of the crack has a tendency to decline.

\subsubsection{A Cracked Specimen of Anti-Plane Problem in Icosahedral QCs Based on FDM}

Anti-plane longitudinal shear problems in icosahedral QCs, the non-zero strain components are $\varepsilon_{y z}=\varepsilon_{z y}, \varepsilon_{z x}=\varepsilon_{x z}, w_{z y}, w_{z x}$. The non-zero stress components are $u_{z}=u_{z}(x, y), w_{z}=w_{z}(x, y)$. The relation of stress and strain based on displacements can be simplified as

$$
\left\{\begin{array}{l}
\sigma_{z x}=\sigma_{x z}=\mu \frac{\partial u_{z}}{\partial x}+R \frac{\partial w_{z}}{\partial x}, H_{z x}=\left(K_{1}-K_{2}\right) \frac{\partial w_{z}}{\partial x}+R \frac{\partial u_{z}}{\partial x} \\
\sigma_{z y}=\sigma_{y z}=\mu \frac{\partial u_{z}}{\partial y}+R \frac{\partial w_{z}}{\partial y}, H_{z y}=\left(K_{1}-K_{2}\right) \frac{\partial w_{z}}{\partial y}+R \frac{\partial u_{z}}{\partial y}
\end{array}\right.
$$


The differential equations of equilibrium for anti-plane problem can be expressed by

$$
\frac{\partial \sigma_{z x}}{\partial x}+\frac{\partial \sigma_{z y}}{\partial y}=\rho \frac{\partial^{2} u_{z}}{\partial t^{2}}, \frac{\partial H_{z x}}{\partial x}+\frac{\partial H_{z y}}{\partial y}=\kappa \frac{\partial w_{z}}{\partial t}
$$

On the basis of Equations (106) and (107) one can get

$$
\mu \nabla^{2} u_{z}+R \nabla^{2} w_{z}=\rho \frac{\partial^{2} u_{z}}{\partial t^{2}}, R \nabla^{2} u_{z}+\left(K_{1}-K_{2}\right) \nabla^{2} w_{z}=\kappa \frac{\partial w_{z}}{\partial t}
$$

If let $c_{1}=\mu / \rho, c_{2}=R / \rho, c_{3}=R / \kappa, c_{4}=\left(K_{1}-K_{2}\right) / \kappa, u_{z}=u, w_{z}=w$, the equations can be expressed by

$$
\frac{\partial^{2} u}{\partial t^{2}}=c_{1} \nabla^{2} u+c_{2} \nabla^{2} w, \frac{\partial w}{\partial t}=c_{3} \nabla^{2} u+c_{4} \nabla^{2} w
$$

where $\sqrt{c_{1}}$ represents the velocity of the phonon field.

Referring to Figure 14, consider an icosahedral QC with a centered crack, the length of which is $2 a$.

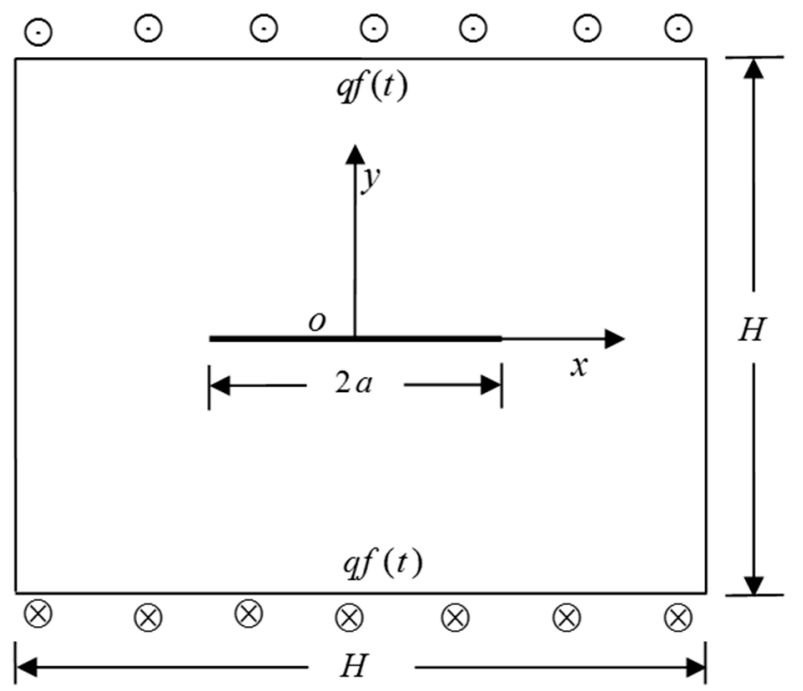

Figure 14. A schematic of a mode III crack penetrates along z-axial direction.

Without loss of generality, we only consider the upper right quarter of the cracked specimen. Due to the lack of the elastic constants of the phason field, we first assume the stress of phason field $H_{z y}=0$ in the boundary. Then the boundary conditions of the phonon and phason fields can be easily written such that

$$
\begin{cases}\sigma_{z y}=q f(t), H_{z y}=0, & y=H,|x| \leq H \\ \sigma_{z x}=0, H_{z x}=0, & x=-H, x=H, 0 \leq y \leq H \\ \sigma_{z y}=0, H_{z y}=0, & |x| \leq a, y=0 \\ u_{z}=0, w_{z}=0, & |x|>a, y=0\end{cases}
$$

The initial conditions including the initial displacements and velocity ofthe phonon field and phason fieldare equal to zeroes, i.e.,

$$
u_{z}(x, y, 0)=0, w_{z}(x, y, 0)=0,\left.\frac{\partial u_{z}}{\partial t}\right|_{t=0}=0
$$

In the following the length of the crack $a=0.2$, and $H=1$, stress load $=10 \mathrm{MPa}$. The elastic constants can be taken as the following Table 2: 
Table 2. The related parameters used in numerical computation $[16,17,21,69,70]$.

\begin{tabular}{ccccc}
\hline$\mu$ & $\boldsymbol{R}$ & $\boldsymbol{K}_{\mathbf{1}}-\boldsymbol{K}_{\mathbf{2}}$ & $\boldsymbol{\kappa}$ & $\boldsymbol{\rho}$ \\
\hline $0.65 \mathrm{~g} / \mathrm{cm} \cdot \mu \mathrm{s}^{2}$ & $0.0066 \mathrm{~g} / \mathrm{cm}^{3} \cdot \mu \mathrm{s}$ & $0.31 \mathrm{~g} / \mathrm{cm} \cdot \mu \mathrm{s}^{2}$ & $1 / 4.8 \times 10^{10} \mathrm{~g} / \mathrm{cm}^{3} \cdot \mu \mathrm{s}$ & $5.1 \mathrm{~g} / \mathrm{cm}^{3}$ \\
\hline
\end{tabular}

This Figure 15 declares that the normalized dynamic stress intensity factor is equal to zero before the longitudinal wave induced by the dynamic loading propagates to the surface of the crack. The time is exactly equal to that of the wave that propagates to the surface of the crack.

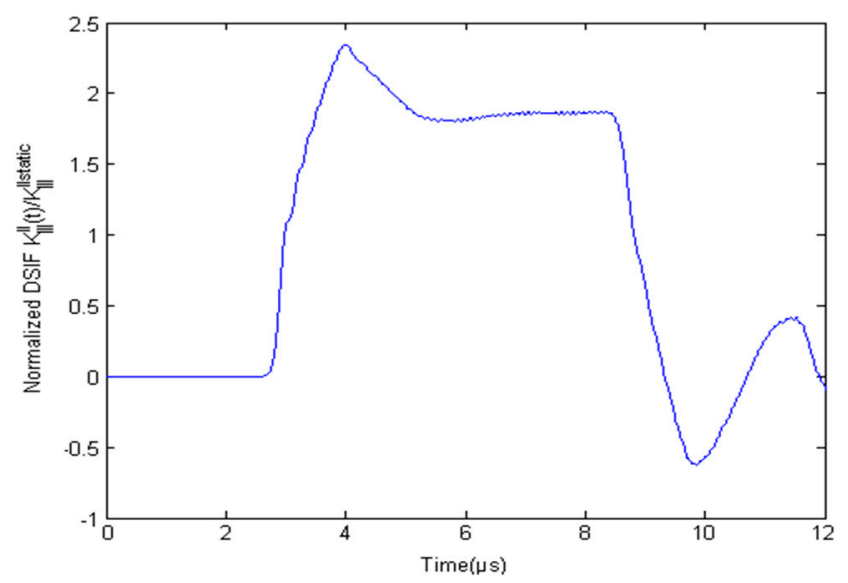

Figure 15. Normalized dynamic stress intensity factor versus time.

From Figure 16, it is obvious that the difference between the displacement of a point at the crack tip of the phonon and phason field is tremendous. The influence of the altered loadings for icosahedral QCs with a crack will be studied below. Three cases are considered. The first one is the load stress is equal to $2 \mathrm{MPa}$. After that the load stress is equal to $2 \mathrm{MPa}$ or $10 \mathrm{MPa}$. The normalized dynamic stress intensity factor (NDSIF) does not alter along with the change of load stress, so the stress at the crack tip increases when the load stress increases. Below, three displacements are depicted in Figures 17 and 18, we can see that, when the load stress increases, the displacements fluctuate drastically.

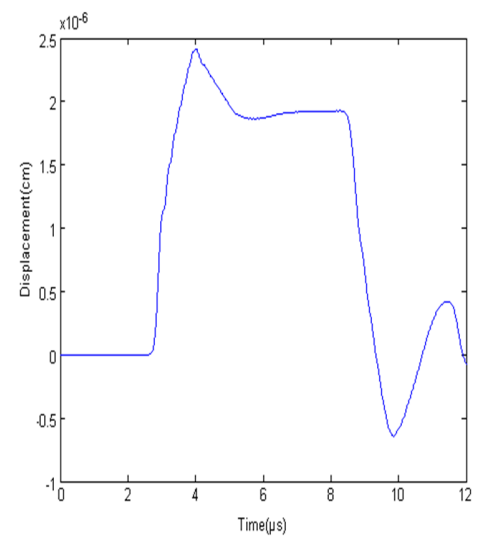

(a)

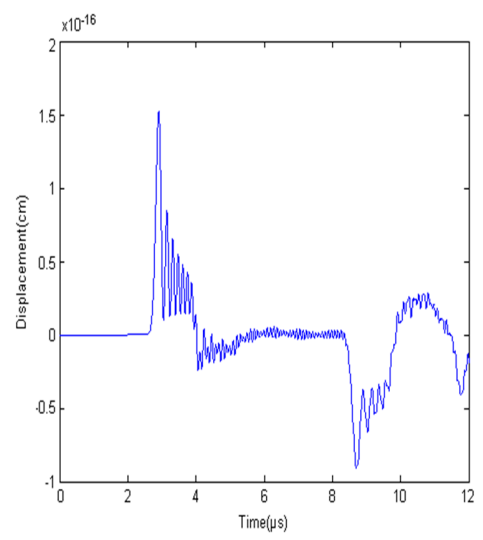

(b)

Figure 16. The displacement of a point at the crack tip of phonon field (a) and phason field (b). 


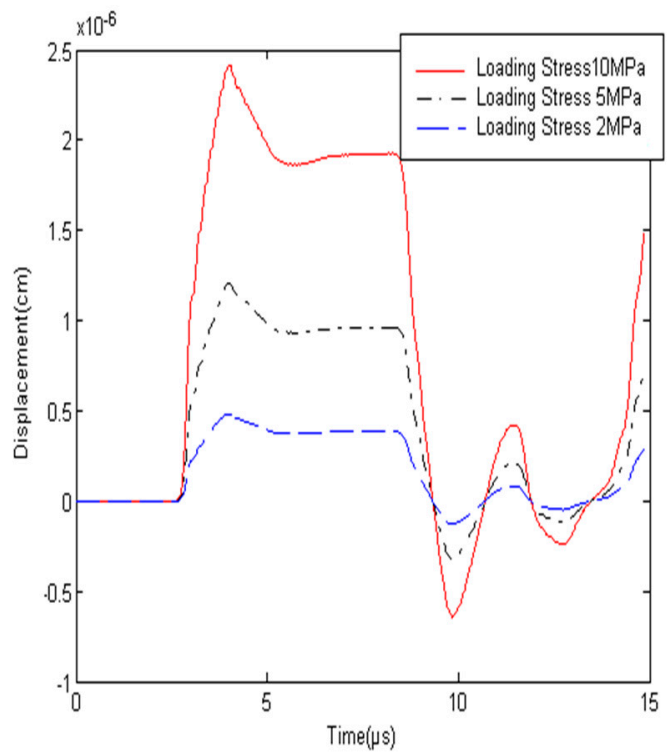

(a)

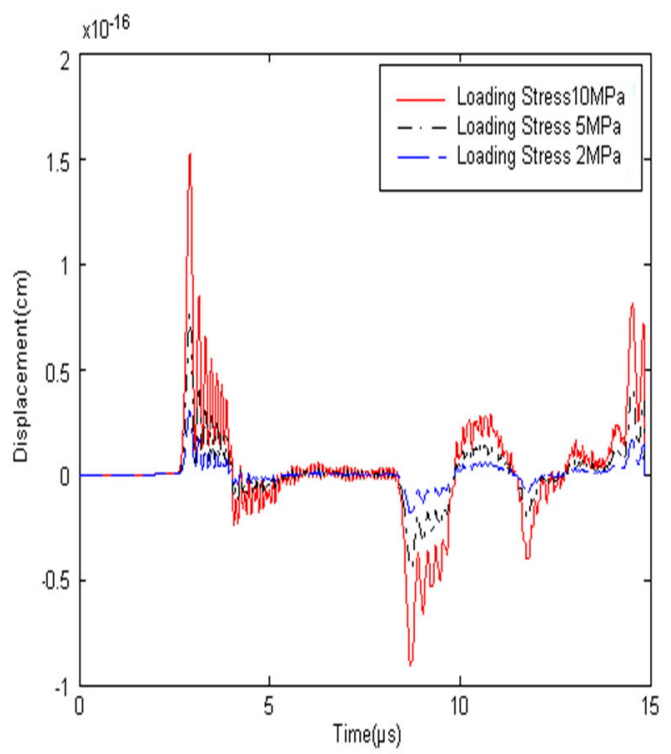

(b)

Figure 17. The displacement of a point at the crack tip of phonon field (a) and phason field (b).

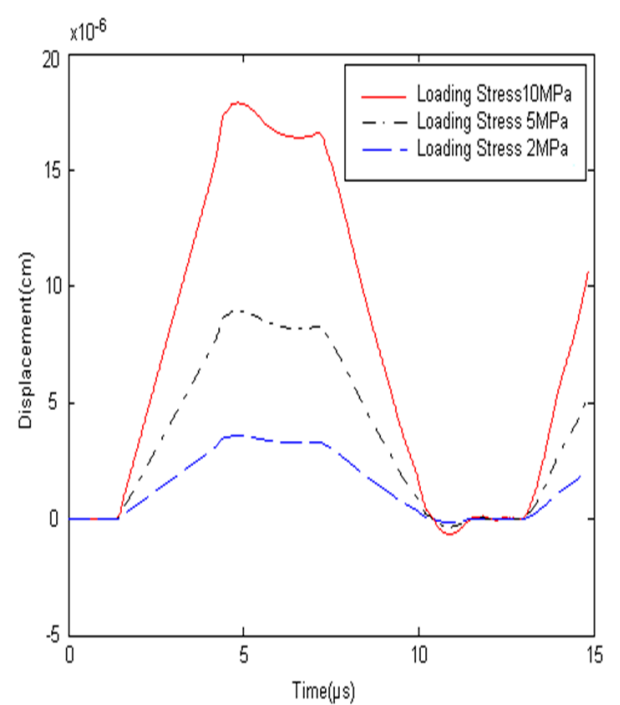

(a)

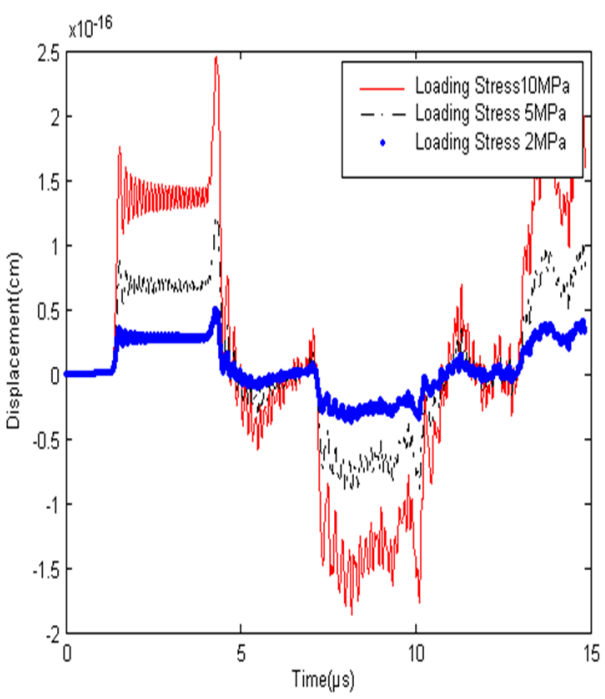

(b)

Figure 18. The displacement of a point at the crack tip of phonon field (a) and phason field (b).

Similar to the plane problem, we apply the stress criterion as the fracture criteria. Assume that the stress value of a point near the crack tip is to control the size of the fracture parameters. Using stress criterion, this paper made a rapidly expanding analysis of the crack. In this problem, three cases will be considered $\sigma_{\text {fractrue }} / \sigma_{\text {initial }}=0.5,0.7,0.9$, in which $\sigma_{\text {fractrue }}$ is the initial fracture stress and $\sigma_{\text {initial }}=100 \mathrm{Mpa}$. The results can be seen in Figure 19. 


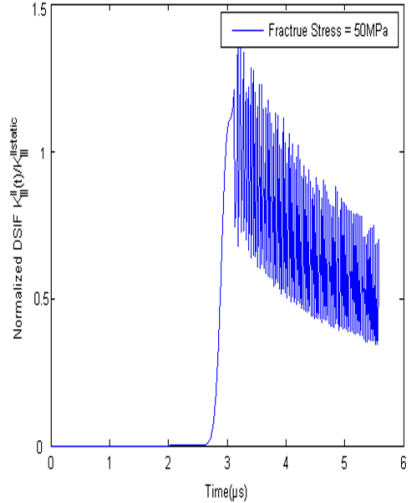

(a)

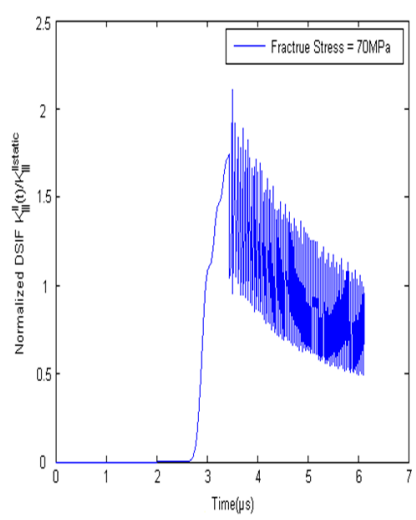

(b)

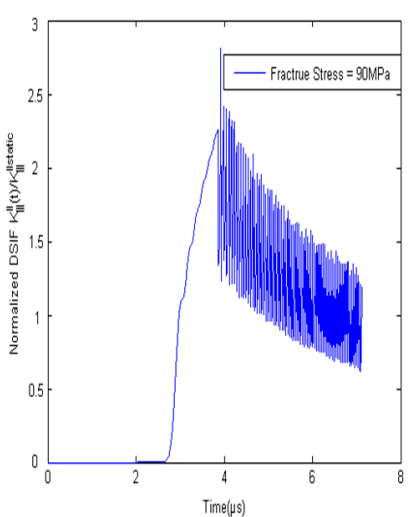

(c)

Figure 19. Normalized dynamic stress intensity factor versus time for $\sigma_{\text {fractrue }}=50 \mathrm{Mpa}$ (a); for $\sigma_{\text {fractrue }}=70 \mathrm{Mpa}(\mathbf{b})$; and for $\sigma_{\text {fractrue }}=90 \mathrm{Mpa}(\mathbf{c})$.

From Figure 20, when the $\sigma_{\text {fractrue }}$ are equal to $50 \mathrm{Mpa}$ and $70 \mathrm{Mpa}$ and $90 \mathrm{Mpa}$, the times of crack extends to the boundary of the material are $5.6 \mu$ s and $6.2 \mu \mathrm{s}$ and $7.2 \mu \mathrm{s}$ respectively. The dynamic stress intensity factors have a uniform change trend based on the different initial fracture stress. When the fracture stress become larger, the situation is different, and the icoshedral QCs (Al-Mn-Pd) start to fracture. The speed of crack propagation increases as the fracture stress reduces, and the fracture resistance of the materials becomes stronger. It is can be inferred that a higher speed of the crack tip can be achieved in the expansion, and the dynamic fracture process corresponds with ordinary materials. It shows that even though the phason field and phonon-phason coupling effects exist in QCs, in the fracture process of QCs and ordinary materials there exist a lot of similarities, and this phenomenon does not go against the basic principle of the fracture dynamics.

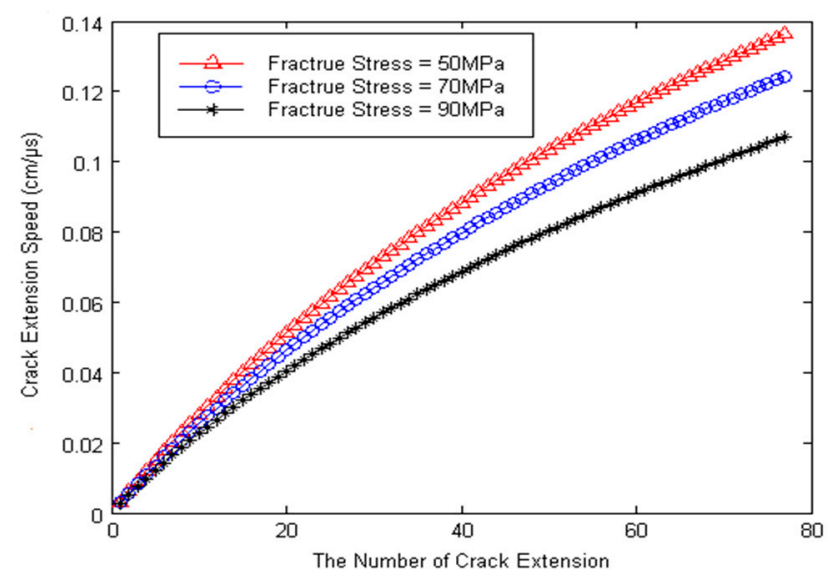

Figure 20. Different speed of extension verses different fracture stress.

\section{Conclusions}

In the previous sections, the dynamics of elasticity and defects of different quasicrystals based on Bak's argument and the argument of Lubensky et al. are formulated and a series of solutions are constructed. The first model can be regarded as elasto-dynamics, and the second one can be regarded as an elasto-/hydro-dynamics model for the material, or as a collaborating model of wave propagation and diffusion. Except for a few examples which are given, some analytic solutions are obtained and a numerical procedure based on the finite difference is used. The finite difference scheme is applied to the dynamic initiation of crack growth and crack fast propagation for two-dimensional decagonal 
$\mathrm{Al}-\mathrm{Ni}$-Co and three-dimensional icosahedral Al-Pd-Mn quasicrystals, the stress intensity factors and displacement and stress fields around the tip of stationary and propagating cracks are investigated.

Acknowledgments: The work is supported by the National Natural Science Foundation of China (No. 11402158) and the Qualified Personnel Foundation of Taiyuan University of Technology (Grant. No. tyut-rc201358a).

Author Contributions: Li Wu and Fan Tianyou prepared the manuscript (text and figures).

Conflicts of Interest: The authors declare no conflict of interest.

\section{References}

1. Shechtman, D.; Blech, I.; Gratias, D.; Cahn, J.W. Metallic phase with long-range orientational order and no translational symmetry. Phys. Rev. Lett. 1984, 53, 1951-1953. [CrossRef]

2. Ohashi, W.; Spaepen, F. Stable Ga-Mg-Zn quasi-periodic crystals with pentagonal dodecahedral solidification morphology. Nature 1987, 330, 555-556. [CrossRef]

3. Wang, N.; Chen, H.; Kuo, K.H. Two-dimensional quasicrystal with eightfold rotational symmetry. Phys. Rev. Lett. 1987, 59, 1010-1013. [CrossRef] [PubMed]

4. Janot, C. The structure of quasicrystals. J. Non-Cryst. Solids 1993, 156-158, 852-864. [CrossRef]

5. Ishimasa, T.; Nissen, H.U. New ordered state between crystalline and am or phous in Ni-Cr particles. Phys. Rev. Lett. 1985, 55, 511-513. [CrossRef] [PubMed]

6. Feng, Y.C.; Lu, G. Experimental evidence for and a projection model of a cubic quasicrystal. J. Phys. Condens. Matter 1990, 2, 9749-9755. [CrossRef]

7. Chen, H.; Li, X.Z.; Zhang, Z.; Kuo, K.H. One-dimensional quasicrystals with twolvefold rotational symmetry. Phys. Rev. Lett. 1998, 60, 1645-1648. [CrossRef] [PubMed]

8. Bohsung, J.; Trebin, H.R. Disclinations in quasicrystals. Phys. Rev. Lett. 1987, 58, 1204-1207. [CrossRef] [PubMed]

9. Ebert, P.H.; Feuerbacher, M.; Tamura, N. Evidence for a cluster-based on structure of Al-Pd-Mn single quasicrystals. Phys. Rev. Lett. 1996, 77, 3827-3830. [CrossRef] [PubMed]

10. Li, C.L.; Liu, Y.Y. Low-temperature lattice excitation of icosahedral Al-Mn-Pd quasicrystals. Phys. Rev. B 2001, 63, 064203. [CrossRef]

11. Rochal, S.B.; Lorman, V.L. Anisotropy of acoustic-phonon properties of an icosahedral quasicrystal at high temperature due to phonon-phason coupling. Phys. Rev. B 2000, 62, 849-874. [CrossRef]

12. Bak, P. Phenomenological theory of icosahedral in commensurate (quasiperiodic) order in $\mathrm{Mn}-\mathrm{Al}$ alloys. Phys. Rev. Lett. 1985, 54, 1517-1519. [CrossRef] [PubMed]

13. Bak, P. Symmetry, stability and elastic properties of icosahedral in commensurate crystals. Phys. Rev. B 1985, 32, 5764-5772. [CrossRef]

14. Socolar, J.E.S.; Lubensky, T.C.; Steinhardt, P.J. Phonons, phasons and dislocations in quasicrystals. Phys. Rev. B 1986, 34, 3345-3360. [CrossRef]

15. Landau, L.D.; Lifshitz, E.M. Statistical Physics; Pergamon Press: Elmsford, NY, USA, 1958.

16. Edagawa, K. Phonon-phason coupling in decagonal quasicrystals. Philos. Mag. 2007, 87, $2789-2798$. [CrossRef]

17. Cheminkov, M.A.; Ott, H.R.; Bianchi, A.; Migliori, A.; Darling, T.W. Elastic moduli of a single quasicrystal of decagonal Al-Ni-Co: Evidence for transverse elastic isotropy. Phys. Rev. Lett. 1998, 80, 321-324.

18. Tanaka, K.; Mitarai, Y.; Koiwa, M. Elastic constants of Al-based icosahedral quasicrystals. Philos. Mag. A 1996, 73, 1715-1723. [CrossRef]

19. Ding, D.H.; Yang, W.G.; Hu, C.Z. Generalized elasticity theory of quasicrystals. Phys. Rev. B 1993, 48, 7003-7010. [CrossRef]

20. Hu, C.Z.; Wang, R.H.; Ding, D.H. Symmetry groups, physical property tensors, elasticity and dislocations in quasicrystals. Rep. Prog. Phys. 2000, 63, 1-39. [CrossRef]

21. Jeong, H.C.; Steinhardt, P.J. Finite-temperature elasticity phase transition in decagonal quasicrystals. Phys. Rev. B 1993, 48, 9394-9403. [CrossRef]

22. Fan, T.Y.; Mai, Y.W. Elasticity theory, fracture mechanics and some relevant thermal properties of quasicrystalline materials. Appl. Mech. Rev. 2004, 57, 325-344. [CrossRef] 
23. Fan, T.Y. Mathematical Theory of Elasticity of Quasicrystals and Its Applications; Springer: Heideberg, Germany, 2010.

24. Levine, D.; Lubensky, T.C.; Ostlund, S. Elasticity and dislocations in pentagonal and icosahedral quasicrystals. Phys. Rev. Lett. 1985, 54, 1520-1523. [CrossRef] [PubMed]

25. Li, X.F.; Duan, X.Y.; Fan, T.Y. Elastic field for a straight dislocation in a decagonal quasicrystal. J. Phys. Condens. Matter 1999, 11, 703-711. [CrossRef]

26. Li, X.F.; Fan, T.Y.; Sun, Y.F. A decagonal quasicrystal with a Griffith crack. Philos. Mag. A 1999, 79, $1943-1952$.

27. Chen, W.Q.; Ma, Y.L.; Ding, H.J. On three-dimensional elastic problems of one dimensional hexagonal quasicrystal bodies. Mech. Res. Commun. 2004, 31, 633-641. [CrossRef]

28. Liu, G.T.; Fan, T.Y.; Guo, R.P. Governing equations and general solutions of plane elasticity of one-dimensional quasicrystals. Int. J. Solids Struct. 2004, 41, 3949-3959. [CrossRef]

29. Li, L.H.; Fan, T.Y. Complex function method for solving notch problem of point 10 two-dimensional quasicrystal based on the stress potential function. J. Phys. Condens. Matter 2006, 18, 10631-10641. [CrossRef]

30. Wang, X.; Pan, E. Analytical solutions for some defect problems in 1D hexagonal and 2D octagonal quasicrystals. Pramana-J. Phys. 2008, 70, 911-933. [CrossRef]

31. Gao, Y.; Xu, S.P.; Zhao, B.S. Boundary conditions for plate bending in one dimensional hexagonal quasicrystals. J. Elast. 2007, 86, 221-233. [CrossRef]

32. Gao, Y.; Ricoeur, A. The refined theory of one-dimensional quasi-crystals inthick plate structures. J. Appl. Mech. 2011, 78, 031021. [CrossRef]

33. Coddens, G. On the problem of the relation between phason elasticity and phason dynamics in quasicrystals. Eur. Phys. J. B 2004, 54, 37-65. [CrossRef]

34. Wang, J.B.; Mancini, L.; Wang, R.H.; Gastaldi, J. Phonon- and phason-type spherical inclusions in icosahedral quasicrystals. J. Phys. Condens. Matter 2003, 15, L363-L370. [CrossRef]

35. Guo, J.H.; Zhang, Z.Y.; Xing, Y.M. Antiplane analysis for an elliptical inclusion in 1D hexagonal piezoelectric quasicrystal composites. Philos. Mag. 2016, 96, 349-369. [CrossRef]

36. Radi, E.; Mariano, P.M. Stationary straight cracks in quasicrystals. Int. J. Fract. 2010, 166, 102-120. [CrossRef]

37. Radi, E.; Mariano, P.M. Steady-state propagation of dislocations in quasi-crystals. Proc. R. Soc. A Math. Phys. 2011, 467, 3490-3508. [CrossRef]

38. Mariano, P.M.; Planas, J. Phason self-actions in quasicrystals. Physica D 2013, 249, 24946-24957. [CrossRef]

39. Lazar, M.; Agiasofitou, E. Fundamentals in generalized elasticity and dislocation theory of quasicrystals: Green tensor, dislocation key-formulas and dislocation loops. Philos. Mag. 2014, 94, 4080-4101. [CrossRef]

40. Li, X.Y. Fundamental solutions of penny-shaped and half infinite plane cracks embedded in an infinite space of one dimensional hexagonal quasi-crystal under thermal loading. Proc. R. Soc. A Math. Phys. 2013, 469, 20130023. [CrossRef]

41. Li, X.Y. Three-dimensional thermo-elastic general solutions of one-dimensional hexagonal quasi-crystal and fundamental solutions. Phys. Lett. A 2012, 376, 2004-2009. [CrossRef]

42. Li, X.Y. Elastic field in an infinite medium of one-dimensional hexagonal quasicrystalwith a planar crack. Int. J. Solids Struct. 2014, 51, 1442-1455. [CrossRef]

43. Sladek, J.; Sladek, V.; Pan, E. Bending analyses of 1D orthorhombic quasicrystal plates. Int. J. Solids Struct. 2013, 50, 3975-3983. [CrossRef]

44. Li, W.; Chai, Y.Z. Anti-plane problem analysis for icosahedral quasicrystals under shear loadings. Chin. Phys. B 2014, 23, 116201. [CrossRef]

45. Wollgarten, M.; Beyss, M.; Urban, K.; Liebertz, H.; Koster, U. Direct evidence for plastic deformation of quasicrystals by means of a dislocationmechanism. Phys. Rev. Lett. 1993, 71, 549-552. [CrossRef] [PubMed]

46. Feuerbacher, M.; Bartsch, M.; Grushko, B.; Messerschmidt, U.; Urban, K. Plastic deformation of decagonal Al-Ni-Co quasicrystals. Philos. Mag. Lett. 1997, 76, 369-376. [CrossRef]

47. Messerschmidt, U.; Bartsch, M.; Feuerbacher, M.; Geyer, B.; Urban, K. Friction mechanism of dislocation motion in icosahedralAl-Pd-Mn quasicrystals. Philos. Mag. A 1999, 79, 2123-2135. [CrossRef]

48. Schall, P.; Feuerbacher, M.; Bartsch, M.; Messerschmidt, U.; Urban, K. Dislocation density evolution upon plastic deformation of Al-Pd-Mn single quasicrystals. Philos. Mag. Lett. 1999, 79, 785-796. [CrossRef]

49. Geyer, B.; Bartsch, M.; Feuerbacher, M.; Urban, K.; Messerschmidt, U. Plastic deformation of icosahedral Al-Pd-Mn single quasicrystals I. Experimental results. Philos. Mag. A 2000, 80, 1151-1163. [CrossRef] 
50. Rosenfeld, R.; Feuerbacher, M. Study of plastically deformed icosahedral Al-Pd-Mn single quasicrystals by transmission electron microscopy. Philos. Mag. Lett. 1995, 72, 375-384. [CrossRef]

51. Caillard, D.; Vanderschaeve, G.; Bresson, L.; Gratias, D. Transmission electron microscopy study of dislocations and extended defects in as-grown icosahedral Al-Pd-Mn single grains. Philos. Mag. A 2000, 80, 237-253. [CrossRef]

52. Lubensky, T.C.; Ramaswamy, S.; Toner, J. Hydrodynamics of icosahedral quasicrystals. Phys. Rev. B 1985, 32, 7444-7452. [CrossRef]

53. Francoual, S.; Livet, F.; de Boissieu, M.; Yakhou, F.; Bley, F.; Letoublon, A.; Caudron, R.; Gastaldi, J. Dynamics of Phason fluctuation in i-Al-Pd-Mn quasicrystals. Phys. Rev. Lett. 2003, 91, 225501. [CrossRef] [PubMed]

54. Rochal, S.B.; Lorman, V.L. Minimal model of the phonon-phason dynamics on icosahedral quasicrystals and its application for the problem of internal friction in the i-Al-Pd-Mn alloys. Phys. Rev. B 2002, 66, 144204. [CrossRef]

55. Mikulla, R.; Stadler, J.; Krul, F.; Trebin, H.R.; Gumbsch, P. Crack propagation in quasicrystals. Phys. Rev. Lett. 1998, 81, 3163-3166. [CrossRef]

56. Takeuchi, S.; Iwanaga, H.; Shibuya, T. Hardness of quasicrystals. Jpn. J. Appl. Phys. 1991, 30, $561-562$. [CrossRef]

57. Rösch, F.; Rudhart, C.; Roth, J.; Trebin, H.R.; Gumbsch, P. Dynamic fracture of icosahedral model quasicrystals: A molecular dynamics study. Phys. Rev. B 2005, 72, 014128. [CrossRef]

58. Shmuely, M.; Alterman, Z.S. Crack propagation analysis by finite differences. J. Appl. Mech. 1973, 40, 902-908. [CrossRef]

59. Murti, V.; Vlliappan, S. The use of quarter point element in dynamic crack analysis. Eng. Fract. Mech. 1982, 23, 585-614. [CrossRef]

60. Fan, T.Y.; Li, X.F.; Sun, Y.F. A moving screw dislocation in one-dimensional hexagonal quasicrystal. Acta Phys. Sin. 1999, 8, 288-295.

61. Fan, T.Y.; Wang, X.F.; Li, W.; Zhu, A.Y. Elasto-hydrodynamics of quasicrystals. Philos. Mag. 2009, 89, 501-512. [CrossRef]

62. Zhu, A.Y.; Fan, T.Y. Dynamic crack propagation in a decagonal Al-Ni-Co quasicrystal. J. Phys. Condens. Matter 2008, 20, 295217. [CrossRef]

63. Wang, X.F.; Fan, T.Y.; Zhu, A.Y. Dynamic behaviour of the icosahedral Al-Pd-Mn quasicrystal with a Griffith crack. Chin. Phys. B 2009, 18, 709-714.

64. Yoffe, E.H. Moving Griffith crack. Philos. Mag. 1951, 43, 739-750. [CrossRef]

65. Tupholme, G.E. An anti-plane shear crack moving in one-dimensional hexagonal quasicrystals. Int. J. Solids Struct. 2015, 71, 255-261. [CrossRef]

66. Li, X.F. A general solution of elasto-hydrodynamics of two dimensionalquasicrystals. Philos. Mag. Lett. 2011, 91, 313-320. [CrossRef]

67. Agiasofitou, E.; Lazar, M. The elastodynamic model of wave-telegraph type for quasicrystals. Int. J. Solids Struct. 2014, 51, 923-929. [CrossRef]

68. Agiasofitou, E.; Lazar, M. On the equations of motion of dislocation in quasicrystals. Mech. Res. Commun. 2014, 57, 27-33. [CrossRef]

69. Amazit, Y.; de Boissieu, M.; Zarembowitch, A. Evidences for elastic isotropy and ultrasonic-attenuation anisotropy in Al-Mn-Pd quasi-crystals. Europhys. Lett. 1992, 20, 703-706. [CrossRef]

70. Takeuchi, S.; Edagawa, K. Chapter 8 of elasticity and plastic properties of quasicrystals. In Handbook of Metal Physics; Elsevier: Amsterdam, The Netherlands, 2007; pp. 267-311.

(C) 2016 by the authors; licensee MDPI, Basel, Switzerland. This article is an open access article distributed under the terms and conditions of the Creative Commons Attribution (CC-BY) license (http://creativecommons.org/licenses/by/4.0/). 